\title{
Equilibrium, uncertainty and risk in hydro-thermal electricity systems*
}

\author{
Andy Philpott ${ }^{\dagger} \quad$ Michael Ferris ${ }^{\ddagger} \quad$ Roger Wets $^{\S}$
}

August 31, 2015

\begin{abstract}
The correspondence of competitive partial equilibrium with a social optimum is well documented in the welfare theorems of economics. These theorems can be applied to single-period electricity pool auctions in which price-taking agents maximize profits at competitive prices, and extend naturally to standard models with locational marginal prices. In hydro-thermal markets where the auctions are repeated over many periods, agents seek to optimize their current and future profit accounting for future prices that depend on uncertain inflows. This makes the agent problems multistage stochastic optimization models, but perfectly competitive partial equilibrium still corresponds to a social optimum when all agents are risk neutral and share common knowledge of the probability distribution governing future inflows. The situation is complicated when agents are risk averse. In this setting we show under mild conditions that a social optimum corresponds to a competitive market equilibrium if agents have time-consistent dynamic coherent risk measures and there are enough traded market instruments to hedge inflow uncertainty. We illustrate some of the consequences of risk aversion on market outcomes using a simple two-stage competitive equilibrium model with three agents.
\end{abstract}

\section{Introduction}

Most industrialised regions of the world have over the last twenty years established wholesale electricity markets that take the form of an auction that matches supply and demand. The exact form of these auction mechanisms vary by jurisdiction, but they typically require offers of energy from suppliers at costs they are willing to supply, and clear a market by dispatching these offers in order of increasing cost. Day-ahead markets such as those implemented in most North American jurisdictions, seek to arrange supply well in advance of its demand, so that thermal units can be prepared in time. Since the demand

*This project has been supported in part by the Air Force Office of Scientific Research, the Department of Energy, the National Science Foundation, and the USDA National Institute of Food and Agriculture.

${ }^{\dagger}$ Electric Power Optimization Centre, University of Auckland, New Zealand.

$\ddagger$ University of Wisconsin, Madison.

$\S$ University of California, Davis. 
cannot be predicted with absolute certainty, these day-ahead markets must be augmented with balancing markets to deal with the variation in load and generator availability in real time.

The market mechanisms are designed to be as efficient as possible in the sense that they should maximize the total welfare of producers and consumers. In a deterministic one-shot setting in which all agents act in perfect competition, the welfare theorems of microeconomics ensure that the auction designs lead to welfare maximization. That welfare maximization can be compromised by the exercise of market power by strategic agents is well known, and many studies (see e.g. [2]) have been carried out to estimate the extent of the inefficiency caused by this.

Market inefficiencies can also be created by uncertainty. Each agent in an electricity market is faced with a dynamic stochastic optimization problem, to maximize their current and (possibly risk-adjusted) future profit. Ideally, an electricity market auction would provide every agent with a stochastic process of electricity prices with which they can perform this optimization, but in practice this is too difficult to arrange. Most markets operate in the short term with a day-ahead auction and a balancing market that is settled in real time. These markets are settled separately which can be inefficient in comparison with a single settlement procedure using stochastic programming, at least when agents act as price takers [11]. In some jurisdictions (like New Zealand) there is no day-ahead market and the market dispatch is computed close to real time for the next trading period and implemented as the day unfolds. Offers of generation and demand forecasts for future periods are used to forecast prices for future periods. These are used to guide each agent in what they offer. In this sense, the auction is iterating towards (but possibly never converging to) a set of prices that represent a realization of the stochastic process that each price-taking agent would want to have at their disposal.

A similar price discovery process occurs on a longer time scale for markets with stored hydro electricity. Generators with hydro-electric reservoirs face an inventory problem. They would like to optimize the release of water from reservoirs to maximize profits using a stochastic process of prices, but this process is not known, and must be deduced by each agent using current and future market conditions and hydrological models of future reservoir inflows. For an agent controlling releases from a hydro-electric reservoir, the marginal cost of supply in the current period involves some modeling of opportunity cost that includes possible high prices in future states of the world with low inflows.

In this paper we study the possible causes of market inefficiency that arise from uncertainty in reservoir inflows. To simplify this analysis, we assume that all agents are price takers who do not act strategically. It is well known that competitive electricity prices for a single trading period and single location can be computed as shadow prices from convex economic dispatch models that maximize total social welfare. These results remain true in the presence of a transmission network as long as the use of transmission assets is appropriately priced [19]. In other words the market must contain enough instruments to price transmission (or equivalently have locational marginal prices). Similarly when hydro reservoirs operated by different agents form a cascade on the same river system, as in the model studied by Lino et al [12], the market needs to be completed by an instrument that allows agents to trade water between reservoirs in order for a competitive equilibrium to correspond to the social optimum. 
A similar incompleteness arises from uncertainty in future inflows. If all agents share the same view about future inflows in the sense that there is a single stochastic process of inflows that is common knowledge, and all agents maximize expected profit using the probability law determining these inflows then a competitive equilibrium will correspond to the welfare-optimizing solution computed by a social planner. If agents have different preferences regarding future inflows and are risk averse then such a welfare result might no longer be true. Indeed it is not clear what risk measure the social planner should use in determining a welfare optimizing solution. This raises the question on how one might complete the market with suitable instruments to enable a welfare maximizing competitive equilibrium.

We provide a partial answer to this problem in the setting where there is a single stochastic process of inflows that is common knowledge, but agents have different attitudes to risk. Following previous work of [10] and [15], we assume that agents are endowed with coherent risk measures as defined by [1]. This means that each agent maximizes their expected profit using a worst-case probability distribution chosen from a well-defined set of distributions that is possibly different for each agent. As shown in [10] and [15], as long as the agents' risk sets intersect, the addition of suitable instruments can provide a complete market for risk that yields a single probability law. In equilibrium, all agents share this law, and so a social planner might compute a welfare maximing solution using a stochastic optimization model.

Our results provide market regulators with a competitive counterfactual model to serve as a benchmark for market prices. A risk-neutral counterfactual solution is likely to incur some energy shortages and corresponding high prices, as these will happen occasionally to minimize the expected cost [14]. To avoid these shortages in the real market, hydro offer prices are often observed to be higher than expected opportunity cost by agents seeking to conserve water. Since these markups can also be interpreted as unilateral exercise of market power by hydro-generators [20], the prices from a risk-averse competitive equilibrium model can provide some indication of the extent of inefficiency of the market, either from incompleteness or exercise of market power.

The paper is laid out as follows. In section 2, to establish notation we present a multistage social planning model, and present the concept of a time-consistent dynamic coherent risk measure. Section 3 restricts this to a two-stage stochastic model with random inflows in the second stage, and presents some example instances of the model with one consumer, one thermal plant and one reservoir. In section 4 , we turn attention to competitive equilibrium under risk and derive the central theorem of the paper that shows how an optimal risk-averse social plan matches competitive equilibrium when agents use dynamic coherent risk measures and markets for risk are complete. Section 5 revisits the examples in section 3 to illustrate the theorem, and we then conclude the paper with some remarks.

\section{Multistage equilibrium}

We begin by defining a risk-averse social planning model for hydroelectric generation. This serves to introduce the modelling framework we will use as well as defining our notation. 


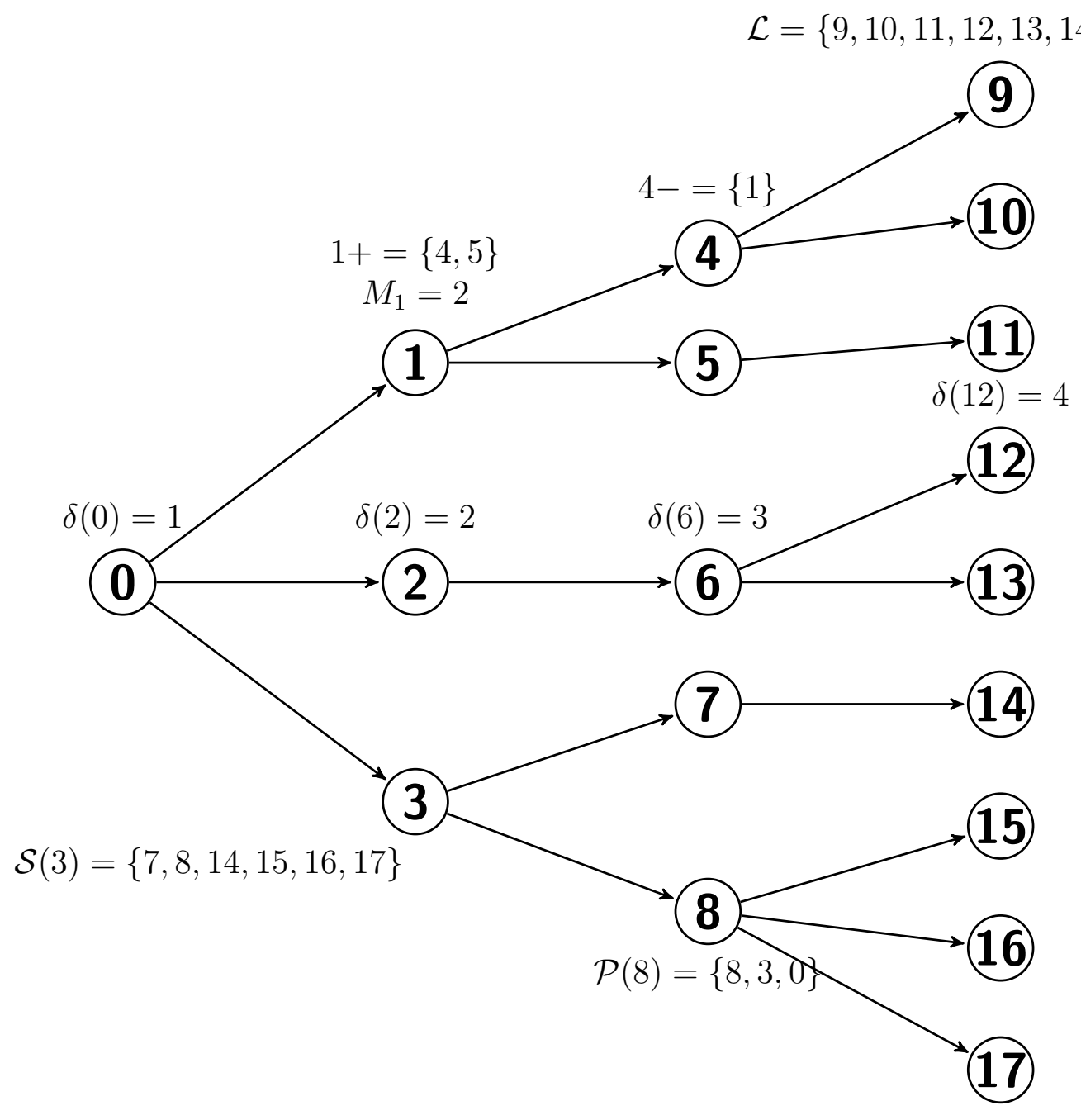

Figure 1: A scenario tree with nodes $\mathcal{N}=\{1,2, \ldots, 17\}$, and $T=4$

We then use a standard Lagrangian decomposition argument to establish a theorem that shows that the optimal risk-averse social plan corresponds to a competitive equilibrium in which each agent maximizes expected profit at equilibrium prices using a risk-adjusted probability measure.

Consider a model of several agents $a \in \mathcal{A}$ assumed to be at the same geographical location (so electricity transmission is ignored). Uncertainty is modeled using a scenario tree with nodes $n \in \mathcal{N}$ and leaves in $\mathcal{L}$. By convention we number the root node $n=0$. The unique predecessor of node $n \neq 0$ is denoted by $n-$. We denote the set of children of node $n \in \mathcal{N} \backslash \mathcal{L}$ by $n+$, and let $M_{n}=\operatorname{card}(n+)$. The set of predecessors of node $n$ on the path from $n$ to node 0 is denoted $\mathcal{P}(n)$ ( so $\mathcal{P}(n)=\{n, n-, n--, \ldots, 0\}$ ), and the set of all successors (not including $n$ ) is denoted $\mathcal{S}(n)\left(\operatorname{so} \mathcal{S}(n)=(n+) \cup_{m \in n+}(m+) \cup \ldots\right)$. The depth $\delta(n)$ of node $n$ is the number of nodes on the path to node 0 , so $\delta(0)=1$ and we assume that every leaf node has the same depth, say $\delta_{\mathcal{L}}$. The depth of a node can be interpreted as a time index $t=1,2, \ldots, T=\delta_{\mathcal{L}}$. A pictorial representation of a scenario tree with 4 time stages is given in Figure 1. 
We denote the actions of agent $a$ in node $n$ by $u_{a}(n)$, defining a stochastic process $\left\{u_{a}(n), n \in \mathcal{N}\right\}$ for agent $a$. We assume that actions might affect later actions through state variables $x_{a}$. Observe that the agents consist of producers and consumers. By convention we assume that $u_{a}(n) \geq 0$ for producers and $u_{a}(n) \leq 0$ for consumers.

We consider a setting in which the electricity generators and consumers are risk averse, and study first a risk-averse social plan, which would be the result of an optimization problem solved by a risk-averse central planner who determines the actions of all agents. Throughout the paper we assume that all agents (and the social planner) use a coherent risk measure as defined by [1] in the context of minimizing some (risk-adjusted) disbenefit function $Z$. A coherent risk measure $\rho(Z)$ has a dual representation expressing it as

$$
\rho(Z)=\sup _{\mu \in \mathcal{D}} \mathbb{E}_{\mu}[Z]
$$

where $\mathcal{D}$ is a convex subset of probability measures (see e.g. $[1,10]$ ). $\mathcal{D}$ is called the risk set of the coherent risk measure. The dual representation using a risk set plays an important role in the analysis we carry out in this paper.

Example 1 Suppose $Z$ represents the random disbenefit of an agent (or social planner), and $\mathrm{AVaR}_{1-\alpha}[Z]$ denotes average value at risk at level $1-\alpha$ that can be expressed as the well-known formula [17]

$$
\mathrm{AVaR}_{1-\alpha}[Z]=\inf _{t}\left\{t+\alpha^{-1} \mathbb{E}\left[(Z-t)_{+}\right]\right\}
$$

Then for any choice of $\lambda \in[0,1]$

$$
\rho(Z)=(1-\lambda) \mathbb{E}[Z]+\lambda \mathrm{AVaR}_{1-\alpha}[Z]
$$

is a coherent risk measure. Here $\lambda=0$ corresponds to a risk-neutral agent and $\lambda=1$ is the most risk averse setting in which all the weight in the objective is placed on $\mathrm{AVaR}_{1-\alpha}[Z]$. If $Z$ has ten equally likely realizations $Z(\omega)$, and $\alpha \leq 0.1$ then the risk set $\mathcal{D}$ is the convex hull of 10 extreme points, where the $k$ th extreme point has probability $\frac{9 \lambda+1}{10}$ for scenario $k$ and $\frac{1-\lambda}{10}$ for the others. Thus

$$
\rho(Z)=\min \left\{\theta: \theta \geq \sum_{\omega=1}^{10} p^{k}(\omega) Z(\omega), \quad k=1,2, \ldots, 10\right\}
$$

where

$$
p^{k}(\omega)=\left\{\begin{array}{cc}
\frac{9 \lambda+1}{10}, & \omega=k \\
\frac{1-\lambda}{10}, & \text { otherwise. }
\end{array}\right.
$$

For a multistage decision problem, we require a dynamic version of risk. The concept of coherent dynamic risk measures was introduced in [16] and is described for general Markov decision problems in [18]. Formally one defines a probability space $(\Omega, \mathcal{F}, P)$ and a filtration $\{\emptyset, \Omega\}=\mathcal{F}_{1} \subset \mathcal{F}_{2} \ldots \subset \mathcal{F}_{T} \subset \mathcal{F}$ of $\sigma$-fields where all data in node 0 is assumed to be deterministic, and decisions at time $t$ are $\mathcal{F}_{t}$-measurable random variables 
(see [18]). Working with finite probability spaces defined by a scenario tree simplifies this description.

Given a tree defined by $\mathcal{N}$, suppose the random sequence of actions $\{u(n), n \in \mathcal{N}\}$ results in a random sequence of costs $\{Z(n), n \in \mathcal{N}\}$. We seek to measure the risk of this cost sequence when viewed by a decision maker at node 0 . At node $n$ the decision maker is endowed with a one-step risk set $\mathcal{D}(n)$ that measures the risk of random risk-adjusted costs accounted for in $m \in n+$. Thus elements of $\mathcal{D}(n)$ are finite probability distributions of the form $\left(p_{1}, p_{2}, \ldots, p_{M_{n}}\right)^{1}$.

The risk-adjusted disbenefit of future outcomes at node $n$ is then defined recursively to be

$$
\begin{array}{rlrl}
\rho_{n}(\{Z(m) & : m \in \mathcal{S}(n)\}) & \\
& = \begin{cases}0, & n \in \mathcal{L}, \\
\sup _{\mu \in \mathcal{D}(n)} \sum_{m \in n+} \mu(m)\left(Z(m)+\rho_{m}(\{Z(q): q \in \mathcal{S}(m)\})\right), & n \in \mathcal{N} \backslash \mathcal{L} .\end{cases}
\end{array}
$$

When viewed in node $n, \rho_{n}$ can be interpreted to be the fair one-time charge we would be willing to incur instead of the sequence of random future costs $\{Z(m): m \in \mathcal{S}(n)\}$. In other words the measure $\rho_{n}$ is a certainty equivalent cost or risk-adjusted expected cost of all the future costs in the subtree rooted at node $n$. As demonstrated in $[18$, Theorem 1], any time-consistent dynamic risk measure has this recursive form.

In the rest of this paper we shall assume that $\mathcal{D}(n)$ is a polyhedron with extreme points $\left(p^{1}(1), p^{2}(2), \ldots, p^{k}\left(M_{n}\right)\right), k \in K(n)$. The recursive structure defined by (2) can then be simplified as follows. Denote the risk-adjusted costs occuring in $m \in n+$ by $\{Z(m)+\theta(m)$, $m \in n+\}$, where $Z(m)$ is the cost in period $\delta(m)$ and $\theta(m)$ is a risk-adjusted future cost. Then

$$
\sup _{\mu \in \mathcal{D}(n)} \sum_{m \in n+} \mu(m)(Z(m)+\theta(m))=\max _{k \in K(n)} \sum_{m \in n+} p^{k}(m)(Z(m)+\theta(m))
$$

since the maximum of a linear function over $\mathcal{D}$ is attained at an extreme point. By a standard dualization, this gives

$$
\begin{aligned}
& \sup _{\mu \in \mathcal{D}(n)} \sum_{m \in n+} \mu(m)(Z(m)+\theta(m)) \\
= & \begin{cases}\min \theta \\
\text { s.t. } & \theta \geq \sum_{m \in n+} p^{k}(m)(Z(m)+\theta(m)), \quad k \in K(n) .\end{cases}
\end{aligned}
$$

Example 2 Suppose that in Figure 1 we have random costs

$$
Z(7)=2 / 3, Z(8)=1, Z(14)=3, Z(15)=5, Z(16)=1, Z(17)=2
$$

and that at every node $n$ we have equal probability to move to any node in $n+$. Also suppose the one-step risk measure at any node $n$ is given by (1) with $\alpha \leq 1 / 3$ and $\lambda=1 / 5$. Then

\footnotetext{
${ }^{1}$ Note that $\mathcal{D}(n)$ in our model is a one-step risk set and does not directly account for the risk of all random sequences of future costs (or their sum over time) conditional on being at node $n$, unless these costs have been converted to some risk-adjusted cost that is added to the period cost.
} 
following the argument of Example 1, we determine $\mathcal{D}(7)=\{1\}$,

$$
\mathcal{D}(3)=\operatorname{conv}\left\{\left(\begin{array}{c}
\frac{3}{5} \\
\frac{2}{5}
\end{array}\right),\left(\begin{array}{c}
\frac{2}{5} \\
\frac{3}{5}
\end{array}\right)\right\}, \mathcal{D}(8)=\operatorname{conv}\left\{\left(\begin{array}{c}
\frac{7}{15} \\
\frac{4}{15} \\
\frac{4}{15}
\end{array}\right),\left(\begin{array}{c}
\frac{4}{15} \\
\frac{7}{15} \\
\frac{4}{15}
\end{array}\right),\left(\begin{array}{c}
\frac{4}{15} \\
\frac{4}{15} \\
\frac{7}{15}
\end{array}\right)\right\} .
$$

Using (2) and (3), it is easy to calculate

$$
\theta(7)=\rho_{7}(\{Z(14)\})=3
$$

and

$$
\theta(8)=\rho_{8}(\{Z(15), Z(16), Z(17)\})=\left(\frac{7}{15}, \frac{4}{15}, \frac{4}{15}\right)\left(\begin{array}{l}
5 \\
1 \\
2
\end{array}\right)=\frac{47}{15} .
$$

Applying the recursive formula at node 3, we compute

$$
\begin{aligned}
\theta(3) & =\rho_{3}(\{Z(7), Z(8), Z(14), Z(15), Z(16), Z(17)\}) \\
& =\max \left\{\left(\frac{3}{5}, \frac{2}{5}\right)\left(\begin{array}{r}
Z(7)+\theta(7) \\
Z(8)+\theta(8)
\end{array}\right),\left(\frac{2}{5}, \frac{3}{5}\right)\left(\begin{array}{c}
Z(7)+\theta(7) \\
Z(8)+\theta(8)
\end{array}\right)\right\} \\
& =\max \left\{\frac{289}{75}, \frac{296}{75}\right\}=\frac{296}{75} .
\end{aligned}
$$

(Note that we can compute this value directly from (1) as

$$
\frac{4}{5} * \frac{1}{2}\left(\frac{11}{3}+\frac{62}{15}\right)+\frac{1}{5} * \frac{62}{15}
$$

using the costs in period 3 plus the risk-adjusted future costs.)

We now turn our attention to a social planning problem that minimizes risk-adjusted generation cost using a social risk measure. At each node $n \in \mathcal{N} \backslash \mathcal{L}$ the social planner uses a risk set $\mathcal{D}_{s}(n)=\operatorname{conv}\left\{\left(p_{s}^{k}(1), p_{s}^{k}(2), \ldots, p_{s}^{k}\left(M_{n}\right)\right), k \in K_{s}(n)\right\}$. Given actions $u_{a}(n)$, $n \in \mathcal{N}$ the social disbenefit in node $n$ is measured by a function $\sum_{a \in \mathcal{A}} C_{a}\left(u_{a}(n)\right)$. Here for generator $a, C_{a}$ measures generation cost, and for consumer $a, C_{a}$ measures consumption disbenefit that increases as $u_{a}$ increases towards 0 . We assume that each $C_{a}$ is convex. The risk-adjusted disbenefit at node 0 is $\sum_{a \in \mathcal{A}} C_{a}\left(u_{a}(0)\right)+\theta(0)$ where $\theta(0)$ is defined recursively by $(2)$, i.e.

$$
\theta(n)= \begin{cases}0, & n \in \mathcal{L} \\ \max _{\mu \in \mathcal{D}_{s}(n)} \sum_{m \in n+} \mu(m)\left(\sum_{a \in \mathcal{A}} C_{a}\left(u_{a}(m)\right)+\theta(m)\right), & n \in \mathcal{N} \backslash \mathcal{L} .\end{cases}
$$

Now using (3) a risk-averse social planning problem can be formulated as the convex 
programming problem

$$
\begin{array}{ll}
\text { SP: } \min & \sum_{a \in \mathcal{A}} C_{a}\left(u_{a}(0)\right)+\theta(0) \\
\text { s.t. } & \theta(n) \geq \sum_{m \in n+} p_{s}^{k}(m)\left(\sum_{a \in \mathcal{A}} C_{a}\left(u_{a}(m)\right)+\theta(m)\right), \\
k \in K_{s}(n), n \in \mathcal{N} \backslash \mathcal{L}, & \\
& x_{a}(n)=x_{a}(n-)-u_{a}(n)+\omega_{a}(n), \quad a \in \mathcal{A}, n \in \mathcal{N}, \\
& \sum_{a \in \mathcal{A}} g_{a}\left(u_{a}(n)\right) \geq 0 \quad n \in \mathcal{N}, \\
& \theta(n)=0, \quad n \in \mathcal{L}, \\
& u_{a}(n) \in \mathcal{U}_{a}, \quad x_{a}(n) \in \mathcal{X}_{a}, \quad n \in \mathcal{N}, \quad a \in \mathcal{A} .
\end{array}
$$

Here we assume that the (given) initial reservoir storage of agent $a$ is denoted $x_{a}(0-)$. The only uncertainty in the model is in future reservoir inflows. The inflow realization $\omega_{a}(n)$ is revealed to the generators at the beginning of stage $\delta(n)$ (before they have determined their actions). If agent $a$ is a hydro generator, $g_{a}(u)$ denotes a concave production function, while $\mathcal{U}_{a}$ and $\mathcal{X}_{a}$ are assumed polyhedral. Without loss of generality, this notation can be extended to other agents in the model SP, namely thermal plant $\left(g_{a}(u)=u, \omega_{a}=0\right.$, $\left.\mathcal{X}_{a}=\mathbb{R}\right)$ and consumers $\left(g_{a}(u)=u, \omega_{a}=0, \mathcal{X}_{a}=\mathbb{R}, \mathcal{U}_{a}=\left\{u_{a} \mid u_{a} \leq 0\right\}\right)$. We shall assume throughout the paper that any nonlinear constraints in SP satisfy a constraint qualification, so SP is equivalent to solving its Karush-Kuhn-Tucker conditions. To keep the choice of the constraint qualification general, we make this a general assumption.

Assumption 1 The nonlinear constraints in SP satisfy a constraint qualification.

Suppose that the optimal solution to SP consists of a set of actions $\left\{u_{a}^{s}(n), a \in \mathcal{A}\right.$, $n \in \mathcal{N}\}$, defining $\left\{x_{a}^{s}(n), a \in \mathcal{A}, n \in \mathcal{N}\right\}$ and $\left\{\theta^{s}(n), \quad n \in \mathcal{N}\right\}$. This gives an optimal risk-adjusted social plan by virtue of the following result.

Lemma 2 The solution $\left\{u_{a}(n), a \in \mathcal{A}, n \in \mathcal{N}\right\},\{\theta(n), \quad n \in \mathcal{N}\}$ to SP satisfies

$$
\theta(n)= \begin{cases}0, & n \in \mathcal{L}, \\ \max _{\mu \in \mathcal{D}_{s}(n)} \sum_{m \in n+} \mu(m)\left(\sum_{a \in \mathcal{A}} C_{a}\left(u_{a}(m)\right)+\theta(m)\right), & n \in \mathcal{N} \backslash \mathcal{L} .\end{cases}
$$

Proof. Suppose $\left\{u_{a}^{s}(n), a \in \mathcal{A}, n \in \mathcal{N}\right\},\left\{\theta^{s}(n), \quad n \in \mathcal{N}\right\}$ solves SP. We show that $\theta^{s}(n)$ satisfies (4). First, for $n \in \mathcal{L}, \theta^{s}(n)=0$. Now suppose (4) is violated for some $\bar{n} \in \mathcal{N} \backslash \mathcal{L}$, i.e. there is some $\varepsilon>0$ such that for every $k \in K_{s}(\bar{n})$

$$
\theta^{s}(\bar{n})-\varepsilon \geq \sum_{m \in \bar{n}+} p_{s}^{k}(m)\left(\sum_{a \in \mathcal{A}} C_{a}\left(u_{a}^{s}(m)\right)+\theta^{s}(m)\right) .
$$


It follows that

$$
\bar{\theta}(n)=\left\{\begin{array}{cc}
\theta^{s}(\bar{n})-\varepsilon, & n=\bar{n} \\
\theta^{s}(n), & \text { otherwise. }
\end{array}\right.
$$

satisfies the constraints of SP. Also if $q=\bar{n}-$, then

$$
\begin{aligned}
& \sum_{n \in q+} p_{s}^{k}(n)\left(\sum_{a \in \mathcal{A}} C_{a}\left(u_{a}^{s}(n)\right)+\bar{\theta}(n)\right) \\
= & \sum_{n \in q+} p_{s}^{k}(n) \sum_{a \in \mathcal{A}} C_{a}\left(u_{a}^{s}(n)\right)+\sum_{n \in q+\backslash\{\bar{n}\}} p_{s}^{k}(n) \theta^{s}(n)+p_{s}^{k}(\bar{n})\left(\theta^{s}(\bar{n})-\varepsilon\right) \\
= & \sum_{n \in q+} p_{s}^{k}(n)\left(\sum_{a \in \mathcal{A}} C_{a}\left(u_{a}^{s}(n)\right)+\theta^{s}(n)\right)-\varepsilon p_{s}^{k}(\bar{n})
\end{aligned}
$$

and

$$
\bar{\theta}(q)=\theta^{s}(q) \geq \sum_{n \in q+} p_{s}^{k}(n)\left(\sum_{a \in \mathcal{A}} C_{a}\left(u_{a}^{s}(n)\right)+\theta^{s}(n)\right), \quad k \in K_{s}(q)
$$

so

$$
\bar{\theta}(q)-\varepsilon p_{s}^{k}(\bar{n}) \geq \sum_{n \in q+} p_{s}^{k}(n)\left(\sum_{a \in \mathcal{A}} C_{a}\left(u_{a}^{s}(n)\right)+\bar{\theta}(n)\right), \quad k \in K_{s}(q) .
$$

Repeating the argument for $q-$ and its parents shows that there is a feasible solution $\left\{u_{a}^{s}(n), a \in \mathcal{A}, n \in \mathcal{N}\right\},\{\bar{\theta}(n), \quad n \in \mathcal{N}\}$ to SP with $\bar{\theta}(0)<\theta^{s}(0)$ contradicting the assumption that $\theta^{s}(n), n \in \mathcal{N}$ solves SP. Thus for every $n \in \mathcal{N} \backslash \mathcal{L}$

$$
\begin{aligned}
\theta^{s}(n) & =\max _{k \in K_{a}(n)} \sum_{m \in n+} p_{s}^{k}(m)\left(\sum_{a \in \mathcal{A}} C_{a}\left(u_{a}^{s}(m)\right)+\theta^{s}(m)\right) \\
& =\max _{\mu \in \mathcal{D}_{a}(n)} \sum_{m \in n+} \mu(m)\left(\sum_{a \in \mathcal{A}} C_{a}\left(u_{a}^{s}(m)\right)+\theta_{a}^{*}(m)\right)
\end{aligned}
$$

showing that $\theta_{a}^{*}(n)$ satisfies (4).

Now consider a competitive equilbrium in a market of risk-neutral agents each of whom maximizes their expected profit assuming a common probability distribution $\sigma(n)$, $n \in \mathcal{N}$. The equilibrium is formally defined by a set of $\operatorname{prices}\{\pi(n), n \in \mathcal{N}\}$, and actions $\{(u(n), x(n), n \in \mathcal{N}\}$ that solve

$$
\begin{aligned}
\operatorname{SPL}(a): \min & \sum_{n \in \mathcal{N}} \sigma(n)\left(C_{a}\left(u_{a}(n)\right)-\pi(n) g_{a}\left(u_{a}(n)\right)\right) \\
\text { s.t. } \quad & x_{a}(n)=x_{a}(n-)-u_{a}(n)+\omega_{a}(n), \quad n \in \mathcal{N}, \\
& u_{a}(n) \in \mathcal{U}_{a}, \quad x_{a}(n) \in \mathcal{X}_{a}, \quad n \in \mathcal{N} .
\end{aligned}
$$

for each agent $a$ and satisfy

$$
0 \leq \sum_{a \in \mathcal{A}} g_{a}\left(u_{a}(n)\right) \perp \pi(n) \geq 0, \quad n \in \mathcal{N} .
$$

We label the MOPEC problem (see Appendix A) defined by the collection of $\operatorname{SPL}(a)$ and (5) as $\mathrm{CE}(\sigma)$. 
Theorem 3 Suppose Assumption 1. If $(u, x, \theta)$ solves SP, then there exists a probability distribution $\sigma(n), n \in \mathcal{N}$ and prices $\pi(n), n \in \mathcal{N}$ so that $(u, x, \pi)$ solves $C E(\sigma)$. That is, the social plan is decomposable as a collection of multi-stage stochastic optimization problems per agent, with coupling via complementarity constraints (5).

Proof. Suppose $(u, x, \theta)$ solves SP. This gives an optimal risk-adjusted social plan by virtue of Lemma 2. The formula (4) yields a collection of "worst case" probability measures $\mu^{*}$, one for each node in $\mathcal{N} \backslash \mathcal{L}$. These measures define conditional probabilities $\mu^{*}(m)$ for each child $m \in n+$, so that

$$
\sum_{m \in n+} \mu^{*}(m)=1 .
$$

If $\mathcal{D}_{s}(n)$ does not lie in the interior of the positive orthant in $\mathbb{R}^{|n+|}$ then it is conceivable that $\mu^{*}(m)=0$ for some $m \in n+$. In this case we can prune $\mathcal{N}$ (by deleting $m$ and all nodes in $\mathcal{S}(m)$ ) so that $\mu^{*}(n)>0$ for every $n \in \mathcal{N} \backslash\{0\}$. Since every node in $\mathcal{N} \backslash \mathcal{L}$ has at least one child $m$ with $\mu^{*}(m)>0$ this pruning retains the property that every leaf node has the same depth. The problem SP has an optimal solution in the pruned tree that matches an optimal solution at the same nodes in the original tree and has the same objective value as SP in the original tree. Therefore, without loss of generality we can assume that for every $n \in \mathcal{N} \backslash\{0\}, \mu^{*}(n)>0$.

Now setting $\mu^{*}(0)=1$, define nodal probabilities

$$
\sigma(n)=\prod_{j \in \mathcal{P}(n)} \mu^{*}(j), \quad n \in \mathcal{N},
$$

with the property that $\sigma(n)>0, n \in \mathcal{N}$. Since SP is a convex program, there exist Lagrange multipliers $\sigma(n) \pi(n)$ for the constraints

$$
\sum_{a \in \mathcal{A}} g_{a}\left(u_{a}(n)\right) \geq 0 \quad n \in \mathcal{N},
$$

(because they satisfy the constraint qualification) so that the optimal solution of SP will minimize the Lagrangian giving

$$
\begin{array}{ll}
\min & \sum_{a \in \mathcal{A}}\left(C_{a}\left(u_{a}(0)\right)-\pi(0) g_{a}\left(u_{a}(0)\right)\right) \\
& +\sum_{m \in 0+} \mu^{*}(m)\left(\sum_{a \in \mathcal{A}}\left(C_{a}\left(u_{a}(m)\right)-\pi(m) g_{a}\left(u_{a}(m)\right)\right)\right. \\
& \left.+\sum_{q \in m+} \mu^{*}(q)\left(\sum_{a \in \mathcal{A}}\left(C_{a}\left(u_{a}(q)\right)-\pi(q) g_{a}\left(u_{a}(q)\right)\right)+(\ldots)\right)\right) \\
\text { s.t. } & x_{a}(n)=x_{a}(n-)-u_{a}(n)+\omega_{a}(n), \quad a \in \mathcal{A}, n \in \mathcal{N}, \\
& u_{a}(n) \in \mathcal{U}_{a}, \quad x_{a}(n) \in \mathcal{X}_{a}, \quad n \in \mathcal{N}, \quad a \in \mathcal{A} .
\end{array}
$$


or

$$
\begin{aligned}
\text { SPL: } \min & \sum_{n \in \mathcal{N}} \sigma(n) \sum_{a \in \mathcal{A}}\left(C_{a}\left(u_{a}(n)\right)-\pi(n) g_{a}\left(u_{a}(n)\right)\right) \\
\text { s.t. } \quad & x_{a}(n)=x_{a}(n-)-u_{a}(n)+\omega_{a}(n), \quad a \in \mathcal{A}, n \in \mathcal{N}, \\
& u_{a}(n) \in \mathcal{U}_{a}, \quad x_{a}(n) \in \mathcal{X}_{a}, \quad n \in \mathcal{N}, \quad a \in \mathcal{A} .
\end{aligned}
$$

SPL decouples into a stochastic optimization problem for each agent $a$, namely

$$
\begin{array}{ll}
\operatorname{SPL}(a): \min & \sum_{n \in \mathcal{N}} \sigma(n)\left(C_{a}\left(u_{a}(n)\right)-\pi(n) g_{a}\left(u_{a}(n)\right)\right) \\
\text { s.t. } \quad & x_{a}(n)=x_{a}(n-)-u_{a}(n)+\omega_{a}(n), \quad n \in \mathcal{N}, \\
& u_{a}(n) \in \mathcal{U}_{a}, \quad x_{a}(n) \in \mathcal{X}_{a}, \quad n \in \mathcal{N} .
\end{array}
$$

Thus if each agent $a$ chooses the social planning solution, and evaluates this using prices $\pi(n), n \in \mathcal{N}$ and probabilities $\sigma(n), n \in \mathcal{N}$, then their actions will optimize $\operatorname{SPL}(a)$. Observe that $\sigma(n)>0$ implies that

$$
0 \leq \sum_{a \in \mathcal{A}} g_{a}\left(u_{a}(n)\right) \perp \pi(n) \geq 0 .
$$

Thus $(u, x, \pi)$ solves $\mathrm{CE}(\sigma)$.

Theorem 3 establishes a correspondence between agent optimization and system optimization that is of limited use. Observe that each agent must maximize their own expected profit using probabilities $\sigma(n), n \in \mathcal{N}$ that are derived from identifying the worst outcomes as measured by social disbenefit. These will correspond to the worst outcomes for each agent only under very special circumstances. In general, agent preferences will differ from the social planner's. To model this, we endow each agent $a \in \mathcal{A}$ with their own polyhedral node-dependent risk set $\mathcal{D}_{a}(n), n \in \mathcal{N} \backslash \mathcal{L}$, where

$$
\mathcal{D}_{a}(n)=\operatorname{conv}\left\{\left(p_{a}^{k}(1), p_{a}^{k}(2), \ldots, p_{a}^{k}\left(M_{n}\right)\right), \quad k \in K_{a}(n)\right\}
$$

where $M_{n}=\operatorname{card}(n+)$ and $K^{a}(n)$ is a finite index set.

In order to get some alignment between the objectives of agents and the social planner, we establish a connection between their risk sets. Henceforth we shall assume that the social planning risk set

$$
\mathcal{D}_{s}(n)=\cap_{a \in \mathcal{A}} \mathcal{D}_{a}(n), n \in \mathcal{N} \backslash \mathcal{L},
$$

which requires the following assumption.

Assumption 4 For every $n \in \mathcal{N} \backslash \mathcal{L}, \cap_{a \in \mathcal{A}} \mathcal{D}_{a}(n) \neq \emptyset$.

Under Assumption 4 we can highlight a special case of Theorem 3.

Corollary 5 If $D_{a}(n)=\left\{\mu_{n}\right\}$ for all a and $n$ then every agent is risk neutral, as is the social planner, since $\mathcal{D}_{s}(n)=\left\{\mu_{n}\right\}$. If $(u, x, \theta)$ solves $S P$ and $\sigma(n) \pi(n) \geq 0$ are the Lagrange multipliers on (6) in $S P$, then $(u, x, \pi)$ solves $C E$. 
When $D_{a}(n)$ is not a singleton for some $a$ and $n$, a social plan using risk sets $\mathcal{D}_{s}(n)$ and a risked competitive equilibrium may not coincide. The latter is defined using the agent problem

$$
\begin{aligned}
& \mathrm{P}(a): \min \quad Z_{a}(0)+\theta_{a}(0) \\
& \text { s.t. } \quad \theta_{a}(n) \geq \sum_{\substack{m \in n+\\
k \in K_{a}(n), n \in \mathcal{N} \backslash \mathcal{L},\\
}} p_{a}^{k}(m)\left(Z_{a}(m)+\theta_{a}(m)\right), \\
& x_{a}(n)=x_{a}(n-)-u_{a}(n)+\omega_{a}(n), \quad n \in \mathcal{N}, \\
& Z_{a}(n)=C_{a}\left(u_{a}(n)\right)-\pi(n) g_{a}\left(u_{a}(n)\right), \quad n \in \mathcal{N}, \\
& \theta_{a}(n)=0, \quad n \in \mathcal{L}, \\
& u_{a}(n) \in \mathcal{U}_{a}, \quad x_{a}(n) \in \mathcal{X}_{a}, \quad n \in \mathcal{N} .
\end{aligned}
$$

Definition 6 A multistage risked equilibrium is a stochastic process of prices $\{\pi(n)$ | $n \in \mathcal{N}\}$, and for each agent $a \in \mathcal{A}$, a corresponding collection of actions, $\left\{u_{a}(n), \theta_{a}(n)\right.$, $n \in \mathcal{N}\}$ with the property that

$$
0 \leq \sum_{a \in \mathcal{A}} g\left(u_{a}(n)\right) \perp \pi(n) \geq 0, n \in \mathcal{N},
$$

and $\left\{u_{a}(n), \theta_{a}(n), n \in \mathcal{N}\right\} \in \arg \max P(a)$.

In general we do not have a guarantee that multi-staged risked equilibrium will exist. In simple examples, we can find them numerically using complementarity software described in Appendix A. In section 4 we examine a model in which agents trade risk to give a competitive equilibrium that corresponds to an optimal solution to SP. When the market for risk in each node $n$ is complete, this gives a methodology of constructing equilibria using optimization.

\section{Two-stage hydro-thermal problems}

To illustrate the results of the previous section, we study a simple two-stage stochastic programming example of hydrothermal scheduling under risk. The scenario tree consists of root node 0 and second stage nodes $m=1,2, \ldots, 10$ with equal probability. We use the risk measure $\rho$ defined in Example 1, namely

$$
\rho(Z)=(1-\lambda) \mathbb{E}[Z]+\lambda \mathrm{AVaR}_{1-\alpha}[Z]
$$

where $Z$ measures disbenefit, $\lambda=0.2$ and $\alpha=0.1$.

The example problem has a hydro-thermal system with one reservoir $(a=1)$, one thermal plant $(a=2)$, and one consumer $(a=3)$. We have

$$
\begin{aligned}
& C_{1}\left(u_{1}\right)=0, \quad g\left(u_{1}\right)=1.5 u_{1}-0.015 u_{1}^{2} \quad u_{1} \in[0,10] \\
& C_{2}\left(u_{2}\right)=u_{2}^{2}, \quad g\left(u_{2}\right)=u_{2} \quad u_{2} \in[0,12] \\
& C_{3}\left(u_{3}\right)=2 u_{3}^{2}+40 u_{3}, \quad g\left(u_{3}\right)=u_{3} \quad u_{3} \in[-\infty, 0]
\end{aligned}
$$


Agent 1 is the only player with reservoir dynamics. In all examples its reservoir has inflow equal to 4 in period 1 (node 0 ), and inflows $\omega(n)=1,2, \ldots, 10$ in period 2 . We assume that reservoir storage $x_{1}$ at the end of the second stage has a value

$$
V\left(x_{1}\right)=10 \log \left(0.1 x_{1}+0.01\right) .
$$

This changes our general formulation in a minor way: the hydro player now incurs a disbenefit of $C_{1}\left(u_{1}(m)\right)-V\left(x_{1}(m)\right)$ in every second stage node. This can be incorporated into our framework with few essential changes.

Risk-neutral case With an initial storage level of 10 units this problem gives the risk-neutral competitive equilibrium shown in Table 1.

\begin{tabular}{cccccccccc} 
stage & $m$ & price & storage & release & thermal & $\begin{array}{r}\text { profit } \\
(\mathrm{T})\end{array}$ & $\begin{array}{r}\text { profit } \\
(\mathrm{H})\end{array}$ & $\begin{array}{r}\text { welfare } \\
(\mathrm{C})\end{array}$ & $\begin{array}{r}\text { welfare } \\
\text { (total) }\end{array}$ \\
\hline 0 & & 1.336 & 7.590 & 6.410 & 0.668 & & & & \\
1 & 1 & 2.539 & 2.865 & 5.725 & 1.269 & 2.057 & 20.417 & 362.283 & 384.758 \\
1 & 2 & 2.053 & 3.590 & 6.000 & 1.027 & 1.500 & 19.418 & 366.863 & 387.781 \\
1 & 3 & 1.696 & 4.387 & 6.203 & 0.848 & 1.165 & 18.809 & 370.264 & 390.238 \\
1 & 4 & 1.431 & 5.236 & 6.355 & 0.716 & 0.958 & 18.514 & 372.809 & 392.281 \\
1 & 5 & 1.231 & 6.121 & 6.470 & 0.616 & 0.825 & 18.445 & 374.746 & 394.016 \\
1 & 6 & 1.076 & 7.031 & 6.559 & 0.538 & 0.735 & 18.529 & 376.252 & 395.516 \\
1 & 7 & 0.953 & 7.961 & 6.629 & 0.477 & 0.673 & 18.716 & 377.446 & 396.835 \\
1 & 8 & 0.855 & 8.904 & 6.686 & 0.427 & 0.629 & 18.969 & 378.411 & 398.008 \\
1 & 9 & 0.774 & 9.857 & 6.733 & 0.387 & 0.596 & 19.264 & 379.204 & 399.064 \\
1 & 10 & 0.706 & 10.818 & 6.772 & 0.353 & 0.571 & 19.585 & 379.866 & 400.022
\end{tabular}

Table 1: Competitive equilibrium (solution to CE) with initial storage of 10.

The social planner's problem that maximizes expected welfare (by minimizing expected generation cost) is shown in Table 2. One can observe that the two solutions are identical, as predicted by Corollary 5.

Risk-aversion We now study each agent's risk-averse optimization problem with given prices $\left(\pi_{1}, \pi_{2}(m)\right)$ when all decision makers use the risk measure

$$
\rho(Z)=0.8 \mathbb{E}[Z]+0.2 \mathrm{AVaR}_{0.9}[Z]
$$

Here each agent's optimization problem can be augmented with a risk term that models their aversion to risk. Observe that such risk aversion will only affect the actions of the hydro agent. The thermal agent and consumer react to the prices they observe in each outcome and minimize their instantaneous disbenefit at these prices. Their optimal actions are thus "wait-and-see" decisions and are not made suboptimal by a change in risk attitude (athough the risked disbenefit arising from these actions will change with choice of risk measure). 


\begin{tabular}{cccccccccc} 
stage & $m$ & price & storage & release & thermal & $\begin{array}{r}\text { profit } \\
(\mathrm{T})\end{array}$ & $\begin{array}{r}\text { profit } \\
(\mathrm{H})\end{array}$ & $\begin{array}{r}\text { welfare } \\
(\mathrm{C})\end{array}$ & $\begin{array}{r}\text { cost } \\
\text { (total) }\end{array}$ \\
\hline 0 & & 1.336 & 7.590 & 6.410 & 0.668 & & & & \\
1 & 1 & 2.539 & 2.865 & 5.725 & 1.269 & 2.057 & 20.417 & 362.283 & -384.758 \\
1 & 2 & 2.053 & 3.590 & 6.000 & 1.027 & 1.500 & 19.418 & 366.863 & -387.781 \\
1 & 3 & 1.696 & 4.387 & 6.203 & 0.848 & 1.165 & 18.809 & 370.264 & -390.238 \\
1 & 4 & 1.431 & 5.236 & 6.355 & 0.716 & 0.958 & 18.514 & 372.809 & -392.281 \\
1 & 5 & 1.231 & 6.121 & 6.470 & 0.616 & 0.825 & 18.445 & 374.746 & -394.016 \\
1 & 6 & 1.076 & 7.031 & 6.559 & 0.538 & 0.735 & 18.529 & 376.252 & -395.516 \\
1 & 7 & 0.953 & 7.961 & 6.629 & 0.477 & 0.673 & 18.716 & 377.446 & -396.835 \\
1 & 8 & 0.855 & 8.904 & 6.686 & 0.427 & 0.629 & 18.969 & 378.411 & -398.008 \\
1 & 9 & 0.774 & 9.857 & 6.733 & 0.387 & 0.596 & 19.264 & 379.204 & -399.064 \\
1 & 10 & 0.706 & 10.818 & 6.772 & 0.353 & 0.571 & 19.585 & 379.866 & -400.022
\end{tabular}

Table 2: Social planning solution (solution to SP) with initial storage of 10. stage $m$ price storage release thermal profit profit welfare cost

\begin{tabular}{rrrrrrrrrr} 
& & \multicolumn{1}{c}{ (T) } & & $(\mathrm{H})$ & $(\mathrm{C})$ & (total) \\
\hline 0 & & 0.816 & 12.291 & 6.709 & 0.408 & & & & \\
1 & 1 & 1.118 & 6.757 & 6.534 & 0.559 & 0.479 & 14.131 & 380.898 & -395.508 \\
1 & 2 & 0.987 & 7.681 & 6.610 & 0.494 & 0.410 & 14.291 & 382.175 & -396.876 \\
1 & 3 & 0.882 & 8.621 & 6.671 & 0.441 & 0.361 & 14.527 & 383.202 & -398.090 \\
1 & 4 & 0.796 & 9.571 & 6.720 & 0.398 & 0.325 & 14.811 & 384.042 & -399.178 \\
1 & 5 & 0.725 & 10.530 & 6.761 & 0.363 & 0.298 & 15.127 & 384.740 & -400.164 \\
1 & 6 & 0.665 & 11.495 & 6.796 & 0.333 & 0.277 & 15.460 & 385.328 & -401.065 \\
1 & 7 & 0.614 & 12.466 & 6.825 & 0.307 & 0.261 & 15.803 & 385.829 & -401.893 \\
1 & 8 & 0.571 & 13.440 & 6.851 & 0.285 & 0.248 & 16.150 & 386.262 & -402.659 \\
1 & 9 & 0.532 & 14.418 & 6.873 & 0.266 & 0.237 & 16.497 & 386.638 & -403.372 \\
1 & 10 & 0.499 & 15.399 & 6.892 & 0.249 & 0.229 & 16.842 & 386.968 & -404.039
\end{tabular}

Table 3: Risk averse social planning solution with initial storage of 15

High initial storage The first example has initial reservoir storage of 15 units. The solution to SP is shown in Table 3 and the competitive equilibrium under risk is shown in Table 4. The solutions as before are identical. Observe that scenario 1 is the worst-case outcome in this example. It leads to the highest system cost, as well as to the lowest profit for the hydro generator and worst welfare for the consumer. The thermal generator has highest profit in scenario 1, but, as shown above it is indifferent to risk in this model as it solves a wait-and-see model.

The risk set of the social planner (with $\lambda=0.2$ ) is

$$
\mathcal{D}=\operatorname{conv}\{(\bar{p}, p, \ldots, p),(p, \bar{p}, \ldots, p), \ldots,(p, p, \ldots, \bar{p})\} .
$$

where $\bar{p}=\frac{9 \lambda+1}{10}=0.28, p=\frac{1-\lambda}{10}=0.08$. Since scenario 1 is the worst case, the risk-averse social planning solution is therefore the same as a risk-neutral social planning solution 


\begin{tabular}{cccccccccc} 
stage & $m$ & price & storage & release & thermal & $\begin{array}{r}\text { profit } \\
(\mathrm{T})\end{array}$ & $\begin{array}{r}\text { profit } \\
(\mathrm{H})\end{array}$ & $\begin{array}{r}\text { welfare } \\
(\mathrm{C})\end{array}$ & $\begin{array}{r}\text { welfare } \\
\text { (total) }\end{array}$ \\
\hline 0 & & 0.816 & 12.291 & 6.709 & 0.408 & & & & \\
1 & 1 & 1.118 & 6.757 & 6.534 & 0.559 & 0.479 & 14.131 & 380.898 & 395.508 \\
1 & 2 & 0.987 & 7.681 & 6.610 & 0.494 & 0.410 & 14.291 & 382.175 & 396.876 \\
1 & 3 & 0.882 & 8.621 & 6.671 & 0.441 & 0.361 & 14.527 & 383.202 & 398.090 \\
1 & 4 & 0.796 & 9.571 & 6.720 & 0.398 & 0.325 & 14.811 & 384.042 & 399.178 \\
1 & 5 & 0.725 & 10.530 & 6.761 & 0.363 & 0.298 & 15.127 & 384.740 & 400.164 \\
1 & 6 & 0.665 & 11.495 & 6.796 & 0.333 & 0.277 & 15.460 & 385.328 & 401.065 \\
1 & 7 & 0.614 & 12.466 & 6.825 & 0.307 & 0.261 & 15.803 & 385.829 & 401.893 \\
1 & 8 & 0.571 & 13.440 & 6.851 & 0.285 & 0.248 & 16.150 & 386.262 & 402.659 \\
1 & 9 & 0.532 & 14.418 & 6.873 & 0.266 & 0.237 & 16.497 & 386.638 & 403.372 \\
1 & 10 & 0.499 & 15.399 & 6.892 & 0.249 & 0.229 & 16.842 & 386.968 & 404.039
\end{tabular}

Table 4: Risk averse competitive equilibrium with initial storage of 15

with adjusted probabilities

$$
(\mu(1), \mu(2), \ldots, \mu(10))=(0.28,0.08, \ldots, 0.08) .
$$

This corresponds to a risk-neutral competitive equilibrium in which all players maximize expected profit assuming these probabilities. The hydro agent will solve $\mathrm{P}(1)$ as defined in (9), the thermal agent will solve $\mathrm{P}(2)$, and the consumer will solve $\mathrm{P}(3)$. Therefore by Corollary 5 we get the same solutions in the social planning solution as we do in the competitive equilibrium, as confirmed by Table 3 and Table 4 .

Low initial storage We now assume an initial storage of 10 units. The risk-neutral results in equilibrium are the same as the social planning solution as predicted by Corollary 5 and demonstrated in Table 1 and Table 2. When we include risk aversion, we obtain the results shown in Table 5 and Table 6 . Table 5 shows a solution to SP when the social

stage $m$ price storage release thermal profit profit welfare cost

\begin{tabular}{rrrrrrrrrr} 
& & & & & & $(\mathrm{T})$ & $(\mathrm{H})$ & $(\mathrm{C})$ & (total) \\
\hline 0 & & 1.545 & 7.710 & 6.290 & 0.773 & & & & \\
1 & 1 & 2.472 & 2.948 & 5.763 & 1.236 & 2.125 & 21.918 & 360.888 & -384.931 \\
1 & 2 & 2.004 & 3.682 & 6.028 & 1.002 & 1.601 & 20.968 & 365.307 & -387.876 \\
1 & 3 & 1.660 & 4.486 & 6.224 & 0.830 & 1.286 & 20.401 & 368.589 & -390.276 \\
1 & 4 & 1.404 & 5.340 & 6.370 & 0.702 & 1.090 & 20.138 & 371.050 & -392.277 \\
1 & 5 & 1.210 & 6.229 & 6.482 & 0.605 & 0.963 & 20.090 & 372.927 & -393.980 \\
1 & 6 & 1.060 & 7.142 & 6.568 & 0.530 & 0.878 & 20.189 & 374.390 & -395.457 \\
1 & 7 & 0.940 & 8.073 & 6.637 & 0.470 & 0.818 & 20.385 & 375.553 & -396.756 \\
1 & 8 & 0.844 & 9.018 & 6.692 & 0.422 & 0.775 & 20.644 & 376.495 & -397.914 \\
1 & 9 & 0.765 & 9.972 & 6.738 & 0.382 & 0.743 & 20.944 & 377.270 & -398.957 \\
1 & 10 & 0.699 & 10.934 & 6.776 & 0.349 & 0.719 & 21.267 & 377.919 & -399.905
\end{tabular}

Table 5: Risk averse social planning solution with initial storage of 10 
planner uses a risk measure $\rho_{0}$ with $\lambda=0.2$, and Table 6 shows the risked competitive equilibrium when all agents use this measure. Observe that the solutions are different.

\begin{tabular}{cccccccccc} 
stage & $m$ & price & storage & release & thermal & $\begin{array}{r}\text { profit } \\
(\mathrm{T})\end{array}$ & $\begin{array}{r}\text { profit } \\
(\mathrm{H})\end{array}$ & $\begin{array}{r}\text { welfare } \\
(\mathrm{C})\end{array}$ & $\begin{array}{r}\text { welfare } \\
\text { (total) }\end{array}$ \\
\hline 0 & & 1.317 & 7.580 & 6.420 & 0.658 & & & & \\
1 & 1 & 2.545 & 2.858 & 5.722 & 1.272 & 2.053 & 20.280 & 362.407 & 384.740 \\
1 & 2 & 2.057 & 3.582 & 5.998 & 1.029 & 1.492 & 19.277 & 367.002 & 387.771 \\
1 & 3 & 1.700 & 4.378 & 6.202 & 0.850 & 1.156 & 18.664 & 370.413 & 390.233 \\
1 & 4 & 1.434 & 5.226 & 6.353 & 0.717 & 0.948 & 18.366 & 372.965 & 392.279 \\
1 & 5 & 1.233 & 6.111 & 6.469 & 0.616 & 0.814 & 18.295 & 374.908 & 394.017 \\
1 & 6 & 1.077 & 7.022 & 6.558 & 0.539 & 0.724 & 18.378 & 376.418 & 395.520 \\
1 & 7 & 0.955 & 7.951 & 6.629 & 0.477 & 0.661 & 18.564 & 377.615 & 396.840 \\
1 & 8 & 0.856 & 8.894 & 6.686 & 0.428 & 0.617 & 18.816 & 378.582 & 398.015 \\
1 & 9 & 0.775 & 9.847 & 6.733 & 0.387 & 0.584 & 19.111 & 379.377 & 399.071 \\
1 & 10 & 0.707 & 10.808 & 6.772 & 0.353 & 0.559 & 19.432 & 380.040 & 400.031
\end{tabular}

Table 6: Risk averse competitive equilibrium with initial storage of 10

Low initial storage - elastic demand The solutions above assume a consumer disbenefit measured by $C_{2}\left(u_{3}\right)=2 u_{3}^{2}+40 u_{3}$ which corresponds to a linear inverse demand function

$$
P(d)=40-4 d \text {. }
$$

We can see the effect of a more elastic inverse demand function by solving SP and CE using $C_{2}\left(u_{3}\right)=0.5 u_{3}^{2}+20 u_{3}$, still with initial storage of 10 . This gives the results shown in Table 7 and Table 8 . Table 7 shows a solution to SP when the social planner uses a risk measure $\rho_{0}$ with $\lambda=0.2$, and Table 8 shows the solution to CE when all agents use this measure.

In these examples the worst case profits for the hydro and thermal producers both occur in scenario 10 when water is plentiful. By releasing large amounts of water the (elastic) price decreases to levels that erode their profits. The consumer welfare is maximized in this scenario. The worst-case overall welfare occurs in scenario 1. Although we do not present the results here, this form of equilibrium is not altered by starting with more storage. In contrast to the less elastic case, the worst profits for the hydro producer are always in scenario 10.

In both examples with low storage one can see that the risk-averse social planning solution and the risk-averse competitive equilibrium are different. The social planning solution has highest system cost in scenario 1. In contrast the lowest hydro profit in the risk-averse competitive equilibrium is in scenario 5 in the inelastic case and scenario 10 in the elastic case. Since the hydro generator and the system do not agree on a worst-case outcome, the probability distributions that correspond to an equivalent risk neutral decision will not be common. This means that the competitive equilibrium differs from the plan maximizing total risk-adjusted welfare. We can attempt to construct some agreement on what would be the worst-case outcome by trading risk. This the subject of the next section. 


\begin{tabular}{ccccccrrrr} 
stage & $m$ & price & storage & release & thermal & $\begin{array}{r}\text { profit } \\
(\mathrm{T})\end{array}$ & $\begin{array}{r}\text { profit } \\
(\mathrm{H})\end{array}$ & $\begin{array}{r}\text { welfare } \\
(\mathrm{C})\end{array}$ & $\begin{array}{r}\text { cost } \\
\text { (total) }\end{array}$ \\
\hline 0 & & 5.637 & 5.598 & 8.402 & 2.819 & & & & \\
1 & 1 & 7.917 & 0.851 & 5.746 & 3.959 & 23.614 & 105.874 & 176.144 & -305.633 \\
1 & 2 & 7.149 & 0.975 & 6.623 & 3.575 & 20.721 & 109.091 & 185.720 & -315.532 \\
1 & 3 & 6.416 & 1.122 & 7.476 & 3.208 & 18.236 & 110.626 & 195.407 & -324.269 \\
1 & 4 & 5.722 & 1.297 & 8.301 & 2.861 & 16.129 & 110.722 & 205.082 & -331.932 \\
1 & 5 & 5.069 & 1.507 & 9.090 & 2.535 & 14.369 & 109.636 & 214.610 & -338.614 \\
1 & 6 & 4.463 & 1.760 & 9.838 & 2.232 & 12.924 & 107.638 & 223.844 & -344.406 \\
1 & 7 & 3.907 & 2.062 & 10.536 & 1.954 & 11.761 & 105.006 & 232.633 & -349.400 \\
1 & 8 & 3.406 & 2.421 & 11.177 & 1.703 & 10.844 & 102.014 & 240.831 & -353.689 \\
1 & 9 & 2.961 & 2.844 & 11.754 & 1.480 & 10.136 & 98.914 & 248.314 & -357.363 \\
1 & 10 & 2.573 & 3.333 & 12.265 & 1.286 & 9.599 & 95.915 & 254.997 & -360.511
\end{tabular}

Table 7: Risk averse social planning solution with elastic demand and initial storage 10

\begin{tabular}{cccccrrrrr} 
stage & $m$ & price & storage & release & thermal & $\begin{array}{r}\text { profit } \\
(\mathrm{T})\end{array}$ & $\begin{array}{r}\text { profit } \\
(\mathrm{H})\end{array}$ & $\begin{array}{r}\text { welfare } \\
(\mathrm{C})\end{array}$ & $\begin{array}{r}\text { welfare } \\
\text { (total) }\end{array}$ \\
\hline 0 & & 5.004 & 4.829 & 9.171 & 2.502 & & & & \\
1 & 1 & 8.530 & 0.770 & 5.060 & 4.265 & 24.448 & 99.555 & 178.230 & 302.233 \\
1 & 2 & 7.736 & 0.878 & 5.951 & 3.868 & 21.222 & 104.221 & 187.644 & 313.087 \\
1 & 3 & 6.976 & 1.007 & 6.823 & 3.488 & 18.426 & 107.028 & 197.256 & 322.710 \\
1 & 4 & 6.252 & 1.160 & 7.670 & 3.126 & 16.031 & 108.208 & 206.951 & 331.190 \\
1 & 5 & 5.567 & 1.342 & 8.487 & 2.783 & 14.006 & 108.007 & 216.605 & 338.619 \\
1 & 6 & 4.925 & 1.562 & 9.267 & 2.462 & 12.322 & 106.686 & 226.079 & 345.086 \\
1 & 7 & 4.330 & 1.825 & 10.004 & 2.165 & 10.946 & 104.516 & 235.223 & 350.685 \\
1 & 8 & 3.786 & 2.140 & 10.690 & 1.893 & 9.843 & 101.777 & 243.888 & 355.508 \\
1 & 9 & 3.298 & 2.513 & 11.316 & 1.649 & 8.978 & 98.739 & 251.930 & 359.646 \\
1 & 10 & 2.866 & 2.951 & 11.878 & 1.433 & 8.312 & 95.645 & 259.234 & 363.191
\end{tabular}

Table 8: Risk averse competitive equilibrium with elastic demand and initial storage 10 


\section{Risk trading with polyhedral risk sets}

We now turn our attention to the situation where agents with polyhedral risk sets can trade financial contracts to reduce their risk. We assume that the risk sets for each agent have nonempty intersection and there are enough financial instruments to trade. For a general multistage convex program, formulated in a scenario tree, we prove that a social planning solution corresponds to a perfectly competitive equilibrium with risk trading. This result is then illustrated on our hydro-thermal optimization example.

The financial instruments that are traded are assumed to take a specific form.

Definition 7 Given any node $n$, an Arrow-Debreu security for node $m \in n+i$ s a contract that charges a price $\mu(m)$ in node $n \in \mathcal{N}$, to receive a payment of 1 in node $m \in n+$.

We assume that at every node $n \in \mathcal{N}$, there is an Arrow-Debreu security for each child node $m \in n+$. In other words the market for risk is complete. To reduce its risk, suppose that each agent $a$ in node $n$ purchases $W_{a}(m)$ Arrow-Debreu securities for node $m \in n+$.

Suppose that the optimal social plan consists of a set of actions $\left\{u_{a}^{s}(n), n \in \mathcal{N}\right\}$, and disbenefits accruing to each agent $a$ in node $n$ denoted $Z_{a}^{s}(n)$, and defined by

$$
Z_{a}^{s}(n)=C_{a}\left(u_{a}^{s}(n)\right)-\pi(n) g_{a}\left(u_{a}^{s}(n)\right) .
$$

Note that by virtue of (5)

$$
\sum_{a \in \mathcal{A}} Z_{a}^{s}(n)=\sum_{a \in \mathcal{A}} C_{a}\left(u_{a}^{s}(n)\right) .
$$

We show that prices for energy and for Arrow-Debreu securities can be found so that the optimal social plan is a competitive equilibrium when risk-averse agents trade energy and risk at these prices. Given disbenefits $Z_{a}^{s}(m), m \in n+$, and future risk-adjusted disbenefits $\theta_{a}^{s}(m), m \in n+$, and $\mu$, agent $a$ in node $n$ seeks a portfolio of Arrow-Debreu securities to solve

$$
\begin{aligned}
\mathrm{RT}(a, n): \min & \theta_{a}+\sum_{m \in n+} \mu(m) W_{a}(m) \\
\text { s.t. } & \theta_{a}+\sum_{m \in n+} p_{a}^{k}(m) W_{a}(m) \\
& \geq \sum_{m \in n+} p_{a}^{k}(m)\left(Z_{a}^{s}(m)+\theta_{a}^{s}(m)\right), \quad k \in K_{a}(n) .
\end{aligned}
$$

The optimal solution $\theta_{a}^{*}, W_{a}^{*}(m)$ for a set of risk prices $\mu$ defines what risk products agent $a$ will buy to improve their risked position. If $\mu$ is chosen suitably then these risk trades will clear the risk market. The purchases can be negative, and in equilibrium we require the trades in these to balance. Thus we require for each $m \in n+, n \in \mathcal{N} \backslash \mathcal{L}$,

$$
0 \leq-\sum_{a \in \mathcal{A}} W_{a}(m) \perp \mu(m) \geq 0
$$

The equilibrium trades in each node $n$ that clear the market for Arrow-Debreu securities 
in that node can be found from solving

$$
\begin{aligned}
\operatorname{RT}(n): \min & \sum_{a \in \mathcal{A}} \theta_{a} \\
\text { s.t. } \quad & \theta_{a}+\sum_{m \in n+} p_{a}^{k}(m) W_{a}(m) \\
& \geq \sum_{m \in n+} p_{a}^{k}(m)\left(Z_{a}^{s}(m)+\theta_{a}^{s}(m)\right), k \in K_{a}(n), a \in \mathcal{A}, \\
& -\sum_{a \in \mathcal{A}} W_{a}(m) \geq 0, m \in n+.
\end{aligned}
$$

The social plan gives a system risk-adjusted disbenefit $\theta^{s}(n)$, which by virtue of $(10)$ is defined by

$$
\theta^{s}(n)= \begin{cases}0, & n \in \mathcal{L}, \\ \max _{\mu \in \mathcal{D}_{s}(n)} \sum_{m \in n+} \mu(m)\left[\sum_{a \in \mathcal{A}} Z_{a}^{s}(m)+\theta^{s}(m)\right], & n \notin \mathcal{L} .\end{cases}
$$

Given each agent's disbenefits $Z_{a}^{s}(n), n \in \mathcal{N}$ from a social plan, we define $\theta_{a}(n)$ to be the risk-adjusted current and future disbenefit of agent $a$ in node $n$ with optimal risk trading in that node. We show how $\theta_{a}(n)$ can be constructed recursively. This makes use of the following result.

Lemma 8 Let $n \in \mathcal{N}$ be given and suppose for each $m \in n+$, that $\sum_{a \in \mathcal{A}} \theta_{a}^{s}(m)=\theta^{s}(m)$. Then there exists a risk price $\mu^{*}$ that solves

$$
\operatorname{DTP}(n): \max _{\mu \in \mathcal{D}_{s}(n)} \quad \sum_{m \in n+} \mu(m)\left(\sum_{a \in \mathcal{A}} Z_{a}^{s}(m)+\theta^{s}(m)\right)
$$

and gives an optimal solution $\left(\theta_{a}^{*}(n), W_{a}^{*}(m)\right)$ to $R T(a, n)$, satisfying $\sum_{a \in \mathcal{A}} \theta_{a}^{*}(n)=\theta^{s}(n)$ and

$$
0 \leq-\sum_{a \in \mathcal{A}} W_{a}^{*}(m) \perp \mu^{*}(m) \geq 0
$$

Proof. Consider the risk trading problem RT $(n)$. The linear programming dual of RT $(n)$ is

$$
\begin{array}{rll}
\operatorname{RTD}(n): \quad & \max \sum_{m \in n+} \sum_{a \in \mathcal{A}}\left(\sum_{k=1}^{K_{a}(n)} \lambda_{a}^{k} p_{a}^{k}(m)\right)\left(Z_{a}^{s}(m)+\theta_{a}^{s}(m)\right) & \\
\text { s.t. } & \sum_{k \in K_{a}(n)} \lambda_{a}^{k} p_{a}^{k}(m)-\mu(m)=0, \quad \mu(m) \geq 0, & m \in n+, a \in \mathcal{A}, \\
& \sum_{k \in K_{a}(n)} \lambda_{a}^{k}=1, \quad \lambda_{a}^{k} \geq 0, & a \in \mathcal{A} .
\end{array}
$$

The feasible region of $\operatorname{RTD}(n)$ is clearly bounded, and nonempty since by assumption 1 , $\cap_{a \in \mathcal{A}} \mathcal{D}_{a}(n) \neq \emptyset$. Thus $\operatorname{RTD}(n)$ has an optimal solution. Recalling $\mathcal{D}_{s}(n)=\cap_{a \in \mathcal{A}} \mathcal{D}_{a}(n)$, $\operatorname{RTD}(n)$ is equivalent to $\operatorname{DTP}(n)$ when $\mu(m)$ is substituted for $\sum_{k \in K_{a}(n)} \lambda_{a}^{k} p_{a}^{k}(m)$, and we recall the hypothesis that $\sum_{a \in \mathcal{A}} \theta_{a}(m)=\theta^{s}(m)$. By the duality theorem of linear programming this means that there exists an optimal solution $\left(\theta_{a}^{*}, W_{a}^{*}\right)$ to $\operatorname{RT}(n)$ with the same value $\sum_{a \in \mathcal{A}} \theta_{a}^{*}$ as $\operatorname{DTP}(n)$, which equals $\theta^{s}(n)$ by $(11)$. 
By complementary slackness the solution $\left(\theta_{a}^{*}, W_{a}^{*}\right)$ to $\mathrm{RT}(n)$ clears the market for risk trading, so for each $m \in n+$,

$$
0 \leq-\sum_{a \in \mathcal{A}} W_{a}^{*}(m) \perp \mu^{*}(m) \geq 0 .
$$

The result now follows from the observation that the $\mathrm{KKT}$ conditions of $\mathrm{RT}(n)$ are precisely equal to the MOPEC formed as the collection of the KKT conditions of RT $(a, n)$ combined with the complementarity constraints (12).

We now define an agent's risk-averse optimization problem including risk trading. Given prices $\pi(n), n \in \mathcal{N}$, this involves optimizing profits evaluated with risk trading and risk-adjusted probabilities. The problem for all agents is:

$$
\begin{array}{ll}
\text { SPMT: } \min & \sum_{a \in \mathcal{A}}\left(Z_{a}(0)+\theta_{a}(0)\right) \\
\text { s.t. } & \theta_{a}(n)+\sum_{k \in K_{a}(n), n \in \mathcal{N} \backslash \mathcal{L}, a \in \mathcal{A} \quad\left[p_{a}^{k}(m) W_{a}(m) \geq \sum_{m \in n+} p_{a}^{k}(m)\left(Z_{a}(m)+\theta_{a}(m)\right),\right.} \\
& -\sum_{a \in \mathcal{A}} W_{a}(n) \geq 0, n \in \mathcal{N} \backslash\{0\} \quad[\sigma(n)] \\
& Z_{a}(n)=C_{a}\left(u_{a}(n)\right)-\pi(n) g_{a}\left(u_{a}(n)\right), n \in \mathcal{N}, a \in \mathcal{A} \\
& x_{a}(n)=x_{a}(n-)-u_{a}(n)+\omega_{a}(n), \quad n \in \mathcal{N}, a \in \mathcal{A} \\
& \theta_{a}(n)=0, \quad n \in \mathcal{L}, a \in \mathcal{A} \\
& u_{a}(n) \in \mathcal{U}, \quad x_{a}(n) \in \mathcal{X}, \quad n \in \mathcal{N}, a \in \mathcal{A},
\end{array}
$$

where the quantities in square parentheses indicate the associated multipliers on those constraints.

The next lemma establishes that an optimal solution to SP yields an optimal solution to each agent's problem when they trade risk.

Lemma 9 If $\left(u_{a}, x_{a}\right), a \in \mathcal{A}$, solves $S P$ with prices $\pi$, then there is a set of risk trades $W_{a}, a \in \mathcal{A}$ such that $\left(u_{a}, x_{a}, W_{a}\right), a \in \mathcal{A}$ solves $S P M T$ and for every $m \in \mathcal{N} \backslash\{0\}$

$$
\sum_{a \in \mathcal{A}} W_{a}(m)=0 .
$$

Proof. Suppose $\left(u_{a}, x_{a}\right), a \in \mathcal{A}$, solves SP. Then by Theorem 3 , there exists $\pi$ and $\mu$ so that $\left(u_{a}, x_{a}\right)$ solves SPL. We first use this solution to construct $W_{a}, a \in \mathcal{A}$. Given $\left(u_{a}, x_{a}\right)$ we can construct the system optimal risk-adjusted future disbenefit using Lemma 2, where we recall that $\sum_{a \in \mathcal{A}} Z_{a}^{s}(m)=\sum_{a \in \mathcal{A}} C_{a}\left(u_{a}(m)\right)$ by $(10)$. Consider any node $n$ that has depth $\delta_{\mathcal{L}}-1$. The solution to SP defines for each agent $a \in \mathcal{A}, x_{a}^{s}(n)$, and $u_{a}^{s}(m), m \in n+$. Since $n+\subseteq \mathcal{L}, \theta^{s}(m)=0, m \in n+$, we let $\theta_{a}^{s}(m)=0, m \in n+$. The risk price $\mu^{*}$ from the risked social plan solves

$$
\operatorname{DTP}(n): \max _{\mu \in \mathcal{D}_{s}(n)} \quad \sum_{m \in n+} \mu(m)\left(\sum_{a \in \mathcal{A}} Z_{a}^{s}(m)+\theta^{s}(m)\right)
$$


and so by Lemma 8 this defines an optimal solution $\left(\theta_{a}(n), W_{a}(m)\right)$ to $\operatorname{RT}(a, n)$ for each agent $a \in \mathcal{A}$, satisfying $\sum_{a \in \mathcal{A}} \theta_{a}(n)=\theta^{s}(n)$ and

$$
0 \leq-\sum_{a \in \mathcal{A}} W_{a}(m) \perp \mu^{*}(m) \geq 0, \quad m \in \mathcal{N} \backslash\{0\} .
$$

Note that using the same argument as in Theorem 3, we may assume without loss of generality that $\mu^{*}(m)>0, m \in \mathcal{N} \backslash\{0\}$ so we can recover (13).

This shows that $\sum_{a \in \mathcal{A}} \theta_{a}(n)=\theta^{s}(n)$ for every $n$ with depth $\delta_{\mathcal{L}}-1$. By induction, Lemma 8 implies for any node $n \in \mathcal{N} \backslash \mathcal{L}$ that the risk price $\mu^{*}$ that solves $\operatorname{DTP}(n)$ gives an optimal solution $\left(\theta_{a}(n), W_{a}(m)\right)$ to $\operatorname{RT}(a, n)$ for each agent $a \in \mathcal{A}$, and satisfies (13).

Now observe that by Assumption 4, given $\mu \in D_{s}(n)$, we can construct $\lambda$ satisfying

$$
\begin{array}{ll}
\sum_{k \in K_{a}(n)} \lambda_{a}^{k}(n)=1, & n \in \mathcal{N} \backslash \mathcal{L}, a \in \mathcal{A}, \\
\sum_{k \in K_{a}(n)} \lambda_{a}^{k}(n) p_{a}^{k}(m)=\mu(m), & n \in \mathcal{N} \backslash \mathcal{L}, m \in n+, a \in \mathcal{A}, \\
\lambda_{a}^{k}(n) \geq 0, \mu(n) \geq 0, & k \in K_{a}(n), n \in \mathcal{N} \backslash \mathcal{L}, a \in \mathcal{A} .
\end{array}
$$

It follows that for fixed $n \in \mathcal{N} \backslash \mathcal{L}$ and $m \in n+$ that

$$
\sum_{k \in K_{a}(n)} \lambda_{a}^{k}(n) p_{a}^{k}(m) \sigma(n)=\mu(m) \sigma(n)=\sigma(m)=\sum_{k \in K_{a}(m)} \lambda_{a}^{k}(m) \sigma(m) .
$$

Also note that for any function $\gamma$ defined over $\mathcal{N}$ it follows that

$$
\sum_{n \in \mathcal{N} \backslash\{0\}} \gamma(n)=\sum_{n \in \mathcal{N} \backslash \mathcal{L}} \sum_{m \in n+} \gamma(m)
$$

a fact that we will use in the sequel without further reference.

Now, since $\left(u_{a}, x_{a}\right), a \in \mathcal{A}$ is an optimal solution of SPL, then it also solves

$$
\begin{array}{ll}
\text { LT: } \min & \sum_{a \in \mathcal{A}} \sum_{n \in \mathcal{N}} \sigma(n) Z_{a}(n) \\
\text { s.t. } & Z_{a}(n)=C_{a}\left(u_{a}(n)\right)-\pi(n) g_{a}\left(u_{a}(n)\right), \quad n \in \mathcal{N}, a \in \mathcal{A} \\
& x_{a}(n)=x_{a}(n-)-u_{a}(n)+\omega_{a}(n), \quad n \in \mathcal{N}, a \in \mathcal{A} \\
& \theta_{a}(n)=0, \quad n \in \mathcal{L}, a \in \mathcal{A}, \\
& u_{a}(n) \in \mathcal{U}, \quad x_{a}(n) \in \mathcal{X}, \quad n \in \mathcal{N}, a \in \mathcal{A} .
\end{array}
$$

Using (14) to make substitutions denoted in \{\} brackets, the following three equations hold:

$$
\begin{aligned}
\sum_{a \in \mathcal{A}} \sum_{n \in \mathcal{N}} \sigma(n) Z_{a}(n) & =\sum_{a \in \mathcal{A}} \sigma(0) Z_{a}(0)+\sum_{a \in \mathcal{A}} \sum_{n \in \mathcal{N} \backslash\{0\}} \sigma(n) Z_{a}(n) \\
& =\sum_{a \in \mathcal{A}} Z_{a}(0)+\sum_{a \in \mathcal{A}} \sum_{n \in \mathcal{N} \backslash \mathcal{L}} \sum_{m \in n+} \sigma(m) Z_{a}(m) \\
& =\sum_{a \in \mathcal{A}} Z_{a}(0)+\sum_{a \in \mathcal{A}} \sum_{n \in \mathcal{N} \backslash \mathcal{L}} \sum_{m \in n+}\left\{\sum_{k \in K_{a}(n)} \lambda_{a}^{k}(n) p_{a}^{k}(m) \sigma(n)\right\} Z_{a}(m)
\end{aligned}
$$


and

$$
\begin{aligned}
0 & =\sum_{a \in \mathcal{A}} \sum_{n \in \mathcal{N} \backslash \mathcal{L}} \sum_{m \in n+} W_{a}(m) \cdot 0 \\
& =\sum_{a \in \mathcal{A}} \sum_{n \in \mathcal{N} \backslash \mathcal{L}} \sum_{m \in n+} W_{a}(m)\left\{\sigma(m)-\sum_{k \in K_{a}(n)} \lambda_{a}^{k}(n) p_{a}^{k}(m) \sigma(n)\right\} \\
& =\sum_{a \in \mathcal{A}} \sum_{n \in \mathcal{N} \backslash\{0\}} W_{a}(n) \sigma(n)-\sum_{a \in \mathcal{A}} \sum_{n \in \mathcal{N} \backslash \mathcal{L}} \sum_{m \in n+} \sum_{k \in K_{a}(n)} W_{a}(m) \lambda_{a}^{k}(n) p_{a}^{k}(m) \sigma(n)
\end{aligned}
$$

and

$$
\begin{aligned}
0= & \sum_{a \in \mathcal{A}} \sum_{n \in \mathcal{N} \backslash \mathcal{L}} \sum_{m \in n+} \theta_{a}(m) \cdot 0+\sum_{a \in \mathcal{A}} \theta_{a}(0) \cdot 0 \\
= & \sum_{a \in \mathcal{A}} \sum_{n \in \mathcal{N} \backslash \mathcal{L}} \sum_{m \in n+} \theta_{a}(m)\left\{\sum_{k \in K_{a}(n)} \lambda_{a}^{k}(n) p_{a}^{k}(m) \sigma(n)-\sum_{k \in K_{a}(m)} \lambda_{a}^{k}(m) \sigma(m)\right\} \\
& +\sum_{a \in \mathcal{A}} \theta_{a}(0)\left\{1-\sum_{k \in K_{a}(0)} \lambda_{a}^{k}(0)\right\} \\
= & \sum_{a \in \mathcal{A}} \sum_{n \in \mathcal{N} \backslash \mathcal{L}} \sum_{k \in K_{a}(n)} \sum_{m \in n+} \theta_{a}(m) \lambda_{a}^{k}(n) p_{a}^{k}(m) \sigma(n)-\sum_{a \in \mathcal{A}} \sum_{n \in \mathcal{N} \backslash\{0\}} \theta_{a}(n) \sum_{k \in K_{a}(n)} \lambda_{a}^{k}(n) \sigma(n) \\
& +\sum_{a \in \mathcal{A}} \theta_{a}(0)-\sum_{a \in \mathcal{A}} \theta_{a}(0) \sum_{k \in K_{a}(0)} \lambda_{a}^{k}(0) \sigma(0) \\
= & \sum_{a \in \mathcal{A}} \sum_{n \in \mathcal{N} \backslash \mathcal{L}} \sum_{k \in K_{a}(n)} \sum_{m \in n+} \theta_{a}(m) \lambda_{a}^{k}(n) p_{a}^{k}(m) \sigma(n)-\sum_{a \in \mathcal{A}} \sum_{n \in \mathcal{N} \backslash \mathcal{L} k \in K_{a}(n)} \theta_{a}(n) \lambda_{a}^{k}(n) \sigma(n) \\
& +\sum_{a \in \mathcal{A}} \theta_{a}(0)
\end{aligned}
$$

where we use $\theta_{a}(n)=0, n \in \mathcal{L}$ to discard $\sum_{a \in \mathcal{A}} \sum_{n \in \mathcal{L}} \sum_{k \in K_{a}(n)} \theta_{a}(n) \lambda_{a}^{k}(n) \sigma(n)$ in the last line. Summing these three equations and rearranging terms we get

$$
\begin{aligned}
\sum_{a \in \mathcal{A}} \sum_{n \in \mathcal{N}} \sigma(n) Z_{a}(n) & =\sum_{a \in \mathcal{A}} Z_{a}(0)+\theta_{a}(0) \\
& +\sum_{a \in \mathcal{A}} \sum_{n \in \mathcal{N} \backslash \mathcal{L}} \sum_{k \in K_{a}(n)}\left(-\left(\theta_{a}(n)+\sum_{m \in n+} p_{a}^{k}(m) W_{a}(m)\right)\right) \lambda_{a}^{k}(n) \sigma(n) \\
& \left.+\sum_{a \in \mathcal{A}} \sum_{n \in \mathcal{N} \backslash \mathcal{L}} \sum_{k \in K_{a}(n)}\left(\sum_{m \in n+} p_{a}^{k}(m)\left(Z_{a}(m)+\theta_{a}(m)\right)\right)\right) \lambda_{a}^{k}(n) \sigma(n) \\
& +\sum_{a \in \mathcal{A}} \sum_{n \in \mathcal{N} \backslash\{0\}} \sigma(n) W_{a}(n) .
\end{aligned}
$$

But this is just the Lagrangian for SPMT and thus $\left(u_{a}, x_{a}, W_{a}\right), a \in \mathcal{A}$ solves SPMT. 
Observe that $\sigma$ are the Lagrange multipliers for the constraint

$$
0 \leq-\sum_{a \in \mathcal{A}} W_{a}(n), \quad n \in \mathcal{N} \backslash\{0\}
$$

This means that the optimal $\sigma$ from the social planning problem decouples SPMT into agent problems

$$
\begin{array}{ll}
\operatorname{SPM}(a): \min \quad & Z_{a}(0)+\theta_{a}(0)+\sum_{n \in \mathcal{N} \backslash\{0\}} W_{a}(n) \sigma(n) \\
\text { s.t. } \quad & \theta_{a}(n)+\sum_{m \in n+} p_{a}^{k}(m) W_{a}(m) \geq \sum_{m \in n+} p_{a}^{k}(m)\left(Z_{a}(m)+\theta_{a}(m)\right), \\
& Z_{a}(n)=K_{a}(n), n \in \mathcal{N} \backslash \mathcal{L}, \\
& x_{a}(n)=x_{a}(n-)-u_{a}(n)+\omega_{a}(n), \quad n \in \mathcal{N}, \\
& \theta_{a}(n)=0, \quad n \in \mathcal{L}, \\
& u_{a}(n) \in \mathcal{U}, \quad x_{a}(n) \in \mathcal{X}, \quad n \in \mathcal{N} .
\end{array}
$$

with the property that $\left(u_{a}, x_{a}, W_{a}, Z_{a}, \theta_{a}\right)$ solves $\operatorname{SPM}(a)$. Moreover the optimal solution of $\operatorname{SPM}(a)$ gives

$$
\theta_{a}(n)=\max _{\nu \in \mathcal{D}_{a}(n)} \sum_{m \in n+} \nu(m)\left(\sum_{a \in \mathcal{A}} Z_{a}^{s}(m)-W_{a}^{s}(m)+\theta_{a}(m)\right), n \in \mathcal{N} \backslash \mathcal{L} .
$$

(Since $x_{a}, Z_{a}$, and $\theta_{a}$ are uniquely defined by $\left(u_{a}, W_{a}\right)$, we may also say equivalently that the actions $\left(u_{a}, W_{a}\right) \in \arg \max \operatorname{SPM}(a)$.)

Definition $10 A$ multistage risk-trading equilibrium is a stochastic process of prices $\{\pi(n): n \in \mathcal{N}\},\{\mu(n): n \in \mathcal{N} \backslash\{0\}\}$, and for each agent a $\in \mathcal{A}$, a corresponding collection of actions, $\left\{u_{a}(n), n \in \mathcal{N}\right\},\left\{W_{a}(n), n \in \mathcal{N} \backslash\{0\}\right\}$ with the property that

$$
\begin{aligned}
& 0 \leq \sum_{a \in \mathcal{A}} g_{a}\left(u_{a}(n)\right) \perp \pi(n) \geq 0, n \in \mathcal{N}, \\
& 0 \leq-\sum_{i \in \mathcal{A}} W_{a}(n) \perp \mu(n) \geq 0, n \in \mathcal{N} \backslash\{0\},
\end{aligned}
$$

and $\left\{u_{a}(n), n \in \mathcal{N}\right\},\left\{W_{a}(n), n \in \mathcal{N} \backslash\{0\}\right\} \in \arg \max S P M(a)$.

Theorem 11 Consider a set of agents $a \in \mathcal{A}$, each endowed with a polyhedral nodedependent risk set $\mathcal{D}_{a}(n), n \in \mathcal{N} \backslash \mathcal{L}$ satisfying Assumption 4. Now let $\left\{u_{a}^{s}(n): n \in\right.$ $\mathcal{N}, a \in \mathcal{A}\}$ be a solution to $S P$ with risk sets $D_{s}(n)=\cap_{a \in \mathcal{A}} \mathcal{D}_{a}(n)$. Suppose this gives rise to $\mu$ and prices $\{\pi(n): n \in \mathcal{N}\}$ where $\pi(n) \sigma(n)$ are the Lagrange multipliers for constraints (6). These prices and quantities form a multistage risk-trading equilibrium in which agent a solves $\operatorname{SPM}(a)$ with a policy defined by $u_{a}(\cdot)$ together with a policy of trading Arrow-Debreu securities defined by $\left\{W_{a}(n), n \in \mathcal{N} \backslash\{0\}\right\}$. 
Proof. By Lemma 9 there is a set of risk trades $W_{a}, a \in \mathcal{A}$ such that $\left\{u_{a}(n), n \in\right.$ $\mathcal{N}\},\left\{W_{a}(n), n \in \mathcal{N} \backslash\{0\}\right\} \in \arg \max \operatorname{SPM}(a), a \in \mathcal{A}$. Since $\left\{u_{a}^{s} \mid a \in \mathcal{A}\right\}$ solves SP,

$$
0 \leq \sum_{a \in \mathcal{A}} g_{a}\left(u_{a}(n)\right) \perp \pi(n) \geq 0, n \in \mathcal{N}
$$

and

$$
\sum_{i \in \mathcal{A}} W_{a}(n)=0, n \in \mathcal{N} \backslash\{0\}
$$

by Lemma 9. Thus $\left\{\left(u_{a}(n), W_{a}(m)\right\}\right.$ defines a multistage risk-trading equilibrium.

Theorem 11 shows that under an assumption of a complete market for risk, we may construct a competitive equilibrium with risk trading from a social planning solution. This entails identifying the sets $\mathcal{D}_{s}(n)$ defined by the intersection of the agents' risk sets. The trading in risk to give this equilbrium is not unique, since if $\left(\theta_{a}, W_{a}(m)\right)$ is feasible for $\operatorname{RT}(a, n)$, then so is $\left(\theta_{a}+1, W_{a}(m)-1\right)$ with the same objective. In other words, if $n \in \mathcal{N} \backslash \mathcal{L}$ then we can add a constant $c$ to every payout from the risk contract in node $m \in n+$, and improve the risked position $\theta$, as long as we pay $c$ back in node $n$ with the contract payment $\sum_{m \in n_{+}} \mu(m) c=c$.

\section{$5 \quad$ Risk trading examples}

The risk-trading analysis of the previous section can be applied to our two-stage example problem. Suppose (in contrast to section 3) that we assume that the thermal generator is risk-neutral (i.e. $\lambda=0$ ). This seems reasonable without risk trading since given electricity prices he will act the same irrespective of his choice of $\lambda$. (The same is true of the consumer, although we need only one risk-neutral agent for the following example.) Any risk neutral agent has risk set

$$
\mathcal{D}_{0}=\{(0.1,0.1, \ldots, 0.1)\}
$$

It follows that the intersection of $\mathcal{D}_{0}$ and the other agent's risk sets (which contain $(0.1,0.1, \ldots, 0.1))$ is $\mathcal{D}_{0}$. A complete market for trading risk will then result in an equilibrium that has risk measure

$$
\sup _{\mu \in \mathcal{D}_{0}} \mathbb{E}_{\mu}\left[\sum_{i=1}^{N} Z_{i}\right]=\mathbb{E}\left[\sum_{i=1}^{N} Z_{i}\right] .
$$

Adding risk trading to the risked competitive equilibrium will then give the optimal riskneutral social planning solution.

To illustrate this, suppose that the initial storage in the hydro reservoir is 10 . Table 9 shows the competitive equilibrium when risk trading is allowed in a complete market. Since the thermal generator is risk neutral, the intersection of risk sets for the agents is the singleton $\{0.1,0.1, \ldots, 0.1\}$. Agents in a competitive equilibrium with risk trading will optimize using the worst-case measure in this set. Thus the solution shown in Table 


\begin{tabular}{cccccccccc} 
stage & $m$ & price & storage & release & thermal & $\begin{array}{r}\text { profit } \\
(\mathrm{T})\end{array}$ & $\begin{array}{r}\text { profit } \\
(\mathrm{H})\end{array}$ & $\begin{array}{r}\text { welfare } \\
(\mathrm{C})\end{array}$ & $\begin{array}{r}\text { welfare } \\
\text { (total) }\end{array}$ \\
\hline 0 & & 1.336 & 7.590 & 6.410 & 0.668 & & & & \\
1 & 1 & 2.539 & 2.865 & 5.725 & 1.269 & -8.123 & 19.067 & 373.814 & 384.758 \\
1 & 2 & 2.053 & 3.590 & 6.000 & 1.027 & -5.100 & 19.067 & 373.814 & 387.781 \\
1 & 3 & 1.696 & 4.387 & 6.203 & 0.848 & -2.643 & 19.067 & 373.814 & 390.238 \\
1 & 4 & 1.431 & 5.236 & 6.355 & 0.716 & -0.600 & 19.067 & 373.814 & 392.281 \\
1 & 5 & 1.231 & 6.121 & 6.470 & 0.616 & 1.135 & 19.067 & 373.814 & 394.016 \\
1 & 6 & 1.076 & 7.031 & 6.559 & 0.538 & 2.635 & 19.067 & 373.814 & 395.516 \\
1 & 7 & 0.953 & 7.961 & 6.629 & 0.477 & 3.954 & 19.067 & 373.814 & 396.835 \\
1 & 8 & 0.855 & 8.904 & 6.686 & 0.427 & 5.127 & 19.067 & 373.814 & 398.008 \\
1 & 9 & 0.774 & 9.857 & 6.733 & 0.387 & 6.183 & 19.067 & 373.814 & 399.064 \\
1 & 10 & 0.706 & 10.818 & 6.772 & 0.353 & 7.142 & 19.067 & 373.814 & 400.023
\end{tabular}

Table 9: Risk averse competitive equilibrium with initial storage of 10 and risk trading with $\lambda=0$ for the thermal generator. The equilibrium price of risk is $\mu(m)=0.1$.

\begin{tabular}{cccccccccc} 
stage & $m$ & price & storage & release & thermal & $\begin{array}{r}\text { profit } \\
(\mathrm{T})\end{array}$ & $\begin{array}{r}\text { profit } \\
(\mathrm{H})\end{array}$ & $\begin{array}{r}\text { welfare } \\
(\mathrm{C})\end{array}$ & $\begin{array}{r}\text { welfare } \\
\text { (total) }\end{array}$ \\
\hline 0 & & 1.336 & 7.590 & 6.410 & 0.668 & & & & \\
1 & 1 & 2.539 & 2.865 & 5.725 & 1.269 & 2.057 & 20.417 & 362.283 & 384.758 \\
1 & 2 & 2.053 & 3.590 & 6.000 & 1.027 & 1.500 & 19.418 & 366.863 & 387.781 \\
1 & 3 & 1.696 & 4.387 & 6.203 & 0.848 & 1.165 & 18.809 & 370.264 & 390.238 \\
1 & 4 & 1.431 & 5.236 & 6.355 & 0.716 & 0.958 & 18.514 & 372.809 & 392.281 \\
1 & 5 & 1.231 & 6.121 & 6.470 & 0.616 & 0.825 & 18.445 & 374.746 & 394.016 \\
1 & 6 & 1.076 & 7.031 & 6.559 & 0.538 & 0.735 & 18.529 & 376.252 & 395.516 \\
1 & 7 & 0.953 & 7.961 & 6.629 & 0.477 & 0.673 & 18.716 & 377.446 & 396.835 \\
1 & 8 & 0.855 & 8.904 & 6.686 & 0.427 & 0.629 & 18.969 & 378.411 & 398.008 \\
1 & 9 & 0.774 & 9.857 & 6.733 & 0.387 & 0.596 & 19.264 & 379.204 & 399.064 \\
1 & 10 & 0.706 & 10.818 & 6.772 & 0.353 & 0.571 & 19.585 & 379.866 & 400.022
\end{tabular}

Table 10: Risk neutral competitive equilibrium with initial storage of 10.

9 is the same as the risk neutral competitive equilibrium shown in Table 1 (which is reproduced for convenience as Table 10).

This result depends on the assumption that $\lambda=0$ for the thermal generator. In section 3 , where risk trading was not included, we argued that the thermal generator solves a wait-and-see optimization problem, and so he is indifferent to the choice of $\lambda$. We can then assume $\lambda=0$ for the thermal generator with no loss in generality. However, once the thermal plant can trade risk the choice of $\lambda$ makes a difference to his actions.

To see this consider the equilibrium with risk trading where both agents choose risk measures with $\lambda=0.2$. The equilibrium solution is shown in Table 11. Observe that the risk-averse competitive equilibrium differs from the risk-neutral social planning solution in Table 10. The thermal agent, faced with the possibility of trading risk, is no longer indifferent to his choice of $\lambda$ and now wishes to reduce his exposure to low profits. In the absence of risk trading he could not change his exposure by any actions at all, and so we 


\begin{tabular}{cccccccccc} 
stage & $m$ & price & storage & release & thermal & $\begin{array}{r}\text { profit } \\
(\mathrm{T})\end{array}$ & $\begin{array}{r}\text { profit } \\
(\mathrm{H})\end{array}$ & $\begin{array}{r}\text { welfare } \\
(\mathrm{C})\end{array}$ & $\begin{array}{r}\text { welfare } \\
\text { (total) }\end{array}$ \\
\hline 0 & & 1.545 & 7.710 & 6.290 & 0.773 & & & & \\
1 & 1 & 2.472 & 2.948 & 5.763 & 1.236 & -1.232 & 18.320 & 367.842 & 384.931 \\
1 & 2 & 2.004 & 3.682 & 6.028 & 1.002 & -0.039 & 19.568 & 368.347 & 387.876 \\
1 & 3 & 1.660 & 4.486 & 6.224 & 0.830 & 0.700 & 20.309 & 369.267 & 390.276 \\
1 & 4 & 1.404 & 5.340 & 6.370 & 0.702 & 1.405 & 21.045 & 369.826 & 392.277 \\
1 & 5 & 1.210 & 6.229 & 6.482 & 0.605 & 1.999 & 21.663 & 370.319 & 393.980 \\
1 & 6 & 1.060 & 7.142 & 6.568 & 0.530 & 2.510 & 22.189 & 370.758 & 395.457 \\
1 & 7 & 0.940 & 8.073 & 6.637 & 0.470 & 2.956 & 22.647 & 371.153 & 396.756 \\
1 & 8 & 0.844 & 9.018 & 6.692 & 0.422 & 3.353 & 23.050 & 371.511 & 397.914 \\
1 & 9 & 0.765 & 9.972 & 6.738 & 0.382 & 3.708 & 23.410 & 371.838 & 398.957 \\
1 & 10 & 0.699 & 10.934 & 6.776 & 0.349 & 4.031 & 23.735 & 372.139 & 399.905
\end{tabular}

Table 11: Risk-averse competitive equilibrium with risk trading.

could assume that he was risk neutral. Now we assume that he is endowed with the same risk measure as the hydro generator. The same observations also hold for the consumer. The intersection of the risk sets of all three agents is non-empty, but is not a singleton: it is the risk set shared by each agent.

The risk trading that occurs is shown in Table 12. Risk trading produces the equilib-

\begin{tabular}{cccccr} 
stage & $m$ & price & $\begin{array}{r}\text { trade } \\
(\mathrm{T})\end{array}$ & $\begin{array}{r}\text { trade } \\
(\mathrm{H})\end{array}$ & $\begin{array}{r}\text { trade } \\
(\mathrm{C})\end{array}$ \\
\hline 0 & & & & & \\
1 & 1 & 0.280 & 1.658 & 0.768 & -2.426 \\
1 & 2 & 0.080 & 3.375 & 2.966 & -6.341 \\
1 & 3 & 0.080 & 4.429 & 4.274 & -8.703 \\
1 & 4 & 0.080 & 5.330 & 5.274 & -10.604 \\
1 & 5 & 0.080 & 6.051 & 5.938 & -11.989 \\
1 & 6 & 0.080 & 6.647 & 6.366 & -13.013 \\
1 & 7 & 0.080 & 7.153 & 6.627 & -13.781 \\
1 & 8 & 0.080 & 7.593 & 6.772 & -14.364 \\
1 & 9 & 0.080 & 7.980 & 6.832 & -14.813 \\
1 & 10 & 0.080 & 8.327 & 6.834 & -15.161
\end{tabular}

Table 12: Risk trading between three agents in equilibrium

rium shown in Table 11 that corresponds to a social planning solution that maximizes total expected profit with the worst-case probability distribution in this risk set. We can verify this by examining the total welfare of all agents in the risk-averse equilibrium. This is shown in the last column of Table 11. The smallest welfare occurs in scenario 1. This is the riskiest from the perspective of all agents objectives summed together, and they trade risk to give a minimum risk solution for the sum of their positions. The probability distribution in the intersection of risk sets that corresponds to this equilibrium assigns 0.28 to scenario 1 and 0.08 to the other scenarios. 
The social planning solution that maximizes total social welfare with this risk set is the same as the the risked equilibrium with risk trading. The risk-neutral social planning solution computed with the probabilities defined by the risk price is shown in Table 13. One can observe that the generation levels are the same in each solution but the agents profits differ from those in the equilibrium with trading. Their total is however the same as the total welfare in the equilibrium with trading. The trades have enabled each agent to use the same risk measure, and agree on a worst-case probability distribution.

\begin{tabular}{cccccccccc} 
stage & $m$ & price & storage & release & thermal & $\begin{array}{r}\text { profit } \\
(\mathrm{T})\end{array}$ & $\begin{array}{r}\text { profit } \\
(\mathrm{H})\end{array}$ & $\begin{array}{r}\text { welfare } \\
(\mathrm{C})\end{array}$ & $\begin{array}{r}\text { cost } \\
\text { (total) }\end{array}$ \\
\hline 0 & & 1.545 & 7.710 & 6.290 & 0.773 & & & & \\
1 & 1 & 2.472 & 2.948 & 5.763 & 1.236 & 2.125 & 21.918 & 360.888 & -384.931 \\
1 & 2 & 2.004 & 3.682 & 6.028 & 1.002 & 1.601 & 20.968 & 365.307 & -387.876 \\
1 & 3 & 1.660 & 4.486 & 6.224 & 0.830 & 1.286 & 20.401 & 368.589 & -390.276 \\
1 & 4 & 1.404 & 5.340 & 6.370 & 0.702 & 1.090 & 20.138 & 371.050 & -392.277 \\
1 & 5 & 1.210 & 6.229 & 6.482 & 0.605 & 0.963 & 20.090 & 372.927 & -393.980 \\
1 & 6 & 1.060 & 7.142 & 6.568 & 0.530 & 0.878 & 20.189 & 374.390 & -395.457 \\
1 & 7 & 0.940 & 8.073 & 6.637 & 0.470 & 0.818 & 20.385 & 375.553 & -396.756 \\
1 & 8 & 0.844 & 9.018 & 6.692 & 0.422 & 0.775 & 20.644 & 376.495 & -397.914 \\
1 & 9 & 0.765 & 9.972 & 6.738 & 0.382 & 0.743 & 20.944 & 377.270 & -398.957 \\
1 & 10 & 0.699 & 10.934 & 6.776 & 0.349 & 0.719 & 21.267 & 377.919 & -399.905
\end{tabular}

Table 13: Risk-neutral social planning solution with probabilities defined by risk prices

\section{Conclusions}

The comparison of competitive market equilibrium with social planning solutions is not straightforward when these markets involve uncertain inflows into hydro reservoirs. Even in the risk-neutral setting we need to ensure that there are sufficiently many traded instruments (pricing water exchanges between reservoirs on the same river chain for example) to make a competitive equilibrium coincide with a social planning solution. We have presented a simple class of models for which this result is true in the risk-neutral case.

In a setting with risk-averse agents, further sets of traded instruments are needed to ensure that a social planning solution and a risked equilibrium coincide. Assuming that in each node of the scenario tree there are sufficient Arrow-Debreu securities to cover the possible future outcomes at the next stage, we can derive a multi-stage competitive equilibrium with different risk trades in each node of the scenario tree from a risk-averse social planning solution in this scenario tree.

Finding the appropriate social planning problem to solve is not straightforward since it requires that the social planner have knowledge of the risk sets of each agent (which is private information). However we show that given this information, the planner can in principle solve a risk-averse dynamic optimization problem with an appropriate coherent risk measure (using e.g. the methods discussed in [13]) to yield a stochastic process of 
energy prices that correspond to the outcomes of a competitive equilibrium with risk trading.

This result raises some interesting questions for regulators who are seeking competitive benchmarks with which to monitor the competiveness of electricity markets with hydroelectric reservoirs. A risk-neutral social planning solution is likely to incur some energy shortages and corresponding high prices, as these will happen occasionally to minimize the expected cost. A hydro-thermal market that avoids these shortages is preferable, but prices in periods without shortages will incur risk premiums that are often attributed to unilateral exercise of market power by hydro-generators. The models we develop in this paper are a first step towards estimating perfectly competitive risk premia for these markets, and will assist regulators to diagnose strategic behaviour by generators.

A further question raised by this work is the effect on hydro-firming investment. This requires high prices in dry periods to cover its long-run marginal cost of supply. This raises the possibility of devising an investment model that incorporates risk premia from a risk-averse competitive hydro-thermal model to cover these costs. This would provide an interesting comparison to observed investment in hydro-firming plant.

Finally we remark that the risk-trading models we solve using optimization assume market completeness, which is unrealistic in practice. However in many circumstances, these models admit equilibrium solutions in incomplete markets (as we have demonstrated in some of the above examples). We have also computed equilibria for incomplete market models in which specific financial instruments (such as contracts for differences) are included. Similar computational experiments have been carried out by [6] for models with thermal plant and two-stage uncertainty. Such incomplete market models are currently of small scale, but we expect that before too long improvements in computation will provide regulators and market analysts with a methodology to test the welfare gains that might be realized by introducing practical hedging instruments into markets in which these are absent.

\section{References}

[1] P. Artzner, F. Delbaen, J.-M. Eber, and D. Heath. Coherent measures of risk. Mathematical Finance, 9:203-228, 1999.

[2] S. Borenstein, J.B. Bushnell, and F.A. Wolak. Measuring market inefficiencies in California's restructured wholesale electricity market. American Economic Review, pages 1376-1405, 2002.

[3] W. Britz, M. C. Ferris, and A. Kuhn. Modeling Water Allocating Institutions based on Multiple Optimization Problems with Equilibrium Constraints. Environmental Modelling and Software, 46:196--207, 2013.

[4] A. Brooke, D. Kendrick, and A. Meeraus. GAMS: A User's Guide. The Scientific Press, South San Francisco, California, 1988. 
[5] S. P. Dirkse and M. C. Ferris. The PATH Solver: A Non-Monotone Stabilization Scheme for Mixed Complementarity Problems. Optimization Methods and Software, 5:123-156, 1995.

[6] A. Ehrenmann and Y. Smeers. Generation capacity expansion in a risky environment: a stochastic equilibrium analysis. Operations Research, 59(6):1332-1346, 2011.

[7] M. C. Ferris, S. P. Dirkse, J.-H. Jagla, and A. Meeraus. An Extended Mathematical Programming Framework. Computers and Chemical Engineering, 33:1973-1982, 2009.

[8] M. C. Ferris and T. S. Munson. Complementarity Problems in GAMS and the PATH Solver. Journal of Economic Dynamics and Control, 24:165-188, 2000.

[9] GAMS Development Corporation. EMP User's Manual, 2009.

[10] D. Heath and H. Ku. Pareto equilibria with coherent measures of risk. Mathematical Finance, 14(2):163-172, 2004.

[11] J. Khazaei, G. Zakeri, and S.S. Oren. Market clearing mechanisms under demand uncertainty. Technical report, Electric Power Optimization Centre, University of Auckland, 2013.

[12] P. Lino, L.A.N. Barroso, M.V.F. Pereira, R. Kelman, and M.H.C. Fampa. Bid-based dispatch of hydrothermal systems in competitive markets. Annals of Operations Research, 120(1):81-97, 2003.

[13] A.B. Philpott, V.L. de Matos, and E.C. Finardi. On solving multistage stochastic programs with coherent risk measures. Operations Research, 2013.

[14] A.B. Philpott and Z. Guan. Models for estimating the performance of electricity markets with hydro-electric reservoir storage. Technical report, Electric Power Optimization Centre, University of Auckland, 2013.

[15] D. Ralph and Y. Smeers. Pricing risk under risk measures: an introduction to stochastic-endogenous equilibria. Technical report, 2011.

[16] F. Riedel. Dynamic coherent risk measures. Stochastic Processes and their Applications, 112(2):185-200, 2004.

[17] R.T. Rockafellar and S. Uryasev. Conditional value-at-risk for general loss distributions. Journal of Banking and Finance, 26:1443-1471, 2002.

[18] A. Ruszczyński. Risk-averse dynamic programming for Markov decision processes. Mathematical Programming, 125:235-261, 2010.

[19] F.C. Schweppe, R.D. Tabors, M.C. Caraminis, and R.E. Bohn. Spot pricing of electricity. Kluwer Academic Publishers, Norwell, MA, 1988.

[20] F.A. Wolak. An assessment of the performance of the New Zealand wholesale electricity market. Report for the New Zealand Commerce Commission, 2009. 


\section{A Extended Mathematical Programming}

All of the computational results in this paper are solved using the Extended Mathematical Programming (EMP) $[9,7,3]$ features of the GAMS modeling system [4]. The EMP framework exists to enable formulations of problems that fall outside the standard framework within the modeling system. A high-level description of these extended models, along with tools to automatically create the different realizations or formulations possible, pass them on to the appropriate solvers, and interpret the results in the context of the original model, makes it possible to model more easily, to conduct experiments with formulations otherwise too time-consuming to consider, and to avoid errors that can make results meaningless or worse.

Multiple Optimization Problems with Equilibrium Constraints (MOPEC) involves a collection of agents $\mathcal{A}$ that determine their decisions $x_{\mathcal{A}}=\left(x_{a}, a \in \mathcal{A}\right)$ by solving, independently, an optimization problem,

$$
x_{a} \in \operatorname{argmax}_{x \in \mathbb{R}^{n_{a}}} f_{a}\left(p, x, x_{-a}\right), \quad a \in \mathcal{A},
$$

where $f_{a}\left(p, \cdot x_{-a}\right): \mathbb{R}^{n_{a}} \rightarrow \overline{\mathbb{R}}=\mathbb{R} \cup\{-\infty,+\infty\}$ is their criterion function, with $x_{-a}=$ $\left(x_{o}, o \in \mathcal{A} \backslash\{a\}\right)$ representing the decision of the other agents and $p \in \mathbb{R}^{d}$ being a parameter that may refer to prices in an economic application, stresses in mechanical systems, and environmental conditions in numerous other applications. This parameter and the decisions $x_{\mathcal{A}}$ satisfy a global equilibrium constraint, formulated as a geometric variational inequality,

$$
F\left(p, x_{\mathcal{A}}\right) \in N_{C}(p)
$$

with $N_{C}(p)$ the normal cone to $C$ at $p$. We refer to (15)-(16) as a MOPEC, whose solution is a pair $\left(p, x_{\mathcal{A}}\right)$ that satisfies the preceding inclusions. Even though (15) omits an explicit expression of constraints on $x_{\mathcal{A}}$, that possibility is handled herein by extendedvalue functions.

EMP provides the ability to describe a variational inequality within a modeling system. We annotate existing equations in the model, detailing which ones provide the function $F$, and which ones are part of the description of the underlying feasible set $C$. Note that there is no requirement that $C$ is polyhedral, and the format generalizes both nonlinear equations and nonlinear complementarity systems. The main formulation of interest here is MOPEC, for example the problem described via (15) and (16). In this setting that variables $x_{a}$ and $p$, and the functions $f_{a}$ and $F$ are defined with the usual model system (along with $C$ being defined by equations defC), but an additional annotation is provided of the form:

equilibrium

$\max f_{-} 1 x_{-} 1$

$\max f_{-} 2 x_{-} 2$

$\ldots$

$\max f_{-} k x_{-} k$

vi F p defC

This describes a problem involving $k$ agents, each of which solve an optimization problem whose objective function involves not only variables $x_{k}$ but also other agents 
variables, and the price vector $p$. Similarly to above, the VI involving $F$ and $p$ nails down the values of $p$. In the GAMS implementation, the VI is converted into its KKT form, and then solved using the PATH solver [5, 8] - this allows problems of hundreds or thousands of variables to be processed.

Other features of EMP include stochastic programming and risk measures, hierarchical optimization, such as bilevel programming, extended nonlinear programming and disjunctive programming. 